NOVA

University of Newcastle Research Online

nova.newcastle.edu.au

Quevedo, Daniel E.; Ahlén, Anders; Johansson, Karl H. "State estimation over sensor networks with correlated wireless fading channels" IEEE Transactions on Automatic Control, Vol. 58, Issue 3, p. 581-593 (2013)

Available from: http://dx.doi.org/10.1109/TAC.2012.2212515

Accessed from: http://hdl.handle.net/1959.13/1053091 


\section{State estimation over sensor networks with correlated wireless fading channels}

by Daniel E. Quevedo, Anders Ahlen and Karl H. Johansson

Copyright (C) 2013 IEEE.

This is an author-prepared version of the article, reprinted IEEE Transactions on Automatic Control Vol. 58, Issue 3, p. 581-593 (2013)

http://dx.doi.org/10.1109/TAC.2012.2212515

This material is posted here with permission of the IEEE. Such permission of the IEEE does not in any way imply IEEE endorsement of any of University of Newcastle's products or services. Internal or personal use of this material is permitted. However, permission to reprint/republish this material for advertising or promotional purposes or for creating new collective works for resale or redistribution must be obtained from the IEEE by writing to pubs-permissions@ieee.org. By choosing to view this document, you agree to all provisions of the copyright laws protecting it. 


\title{
State Estimation over Sensor Networks with Correlated Wireless Fading Channels
}

\author{
Daniel E. Quevedo, Member, IEEE, Anders Ahlén, Senior Member, IEEE, \\ and Karl H. Johansson, Senior Member, IEEE
}

\begin{abstract}
Stochastic stability for centralized time-varying Kalman filtering over a wireless sensor network with correlated fading channels is studied. On their route to the gateway, sensor packets, possibly aggregated with measurements from several nodes, may be dropped because of fading links. To study this situation, we introduce a network state process, which describes a finite set of configurations of the radio environment. The network state characterizes the channel gain distributions of the links, which are allowed to be correlated between each other. Temporal correlations of channel gains are modeled by allowing the network state process to form a (semi-)Markov chain. We establish sufficient conditions that ensure the Kalman filter to be exponentially bounded. In the one-sensor case, this new stability condition is shown to include previous results obtained in the literature as special cases. The results also hold when using power and bit-rate control policies, where the transmission power and bit-rate of each node are nonlinear mapping of the network state and channel gains.
\end{abstract}

Index Terms-Sensor networks, Kalman filtering, Packet drops, stability.

\section{INTRODUCTION}

Wireless sensor technology is of growing interest for process and automation industry. The driving force behind using wireless technology in monitoring and control applications is its lower deployment and reconfiguration cost. In addition, wireless devices can be placed where wires cannot go, or where power sockets are not available; see, e.g., [2]-[6].

A drawback of wireless communication technology lies in that wireless channels are subject to fading and interference, which frequently lead to packet errors. The wireless channel is in general time varying. This time-variability may in an industrial setting be caused by moving machines, vehicles, people, and so forth, or when the receiver or the transmitter are mounted on a moving object. Therefore, in addition to the propagation path loss, channels will commonly be subject to shadow and small-scale fading [7], [8]. The channel fading can be partially compensated for through control of bit-rates and the power levels used by the radio amplifiers; see, e.g., [9][12]. The loss of information due to channel fading is one of

Daniel Quevedo is with the School of Electrical Engineering \& Computer Science, The University of Newcastle, NSW 2308, Australia; dquevedo@ieee.org. Anders Ahlén is with Signals and Systems, Uppsala University, SE-751 21, Uppsala, Sweden; Anders.Ahlen@ signal.uu.se. Karl H. Johansson is with ACCESS Linnaeus Centre, School of Electrical Engineering, Royal Institute of Technology, Stockholm, Sweden; kallej@ee.kth.se

This research was supported in part under Australian Research Council's Discovery Projects funding scheme (project number DP0988601), and by the VINNOVA project WiComPI, project Dnr2009-02963. A preliminary version of parts of this work was presented as [1]. the main problems of wireless estimation and control systems, leading to stability and performance degradation.

Several interesting approaches have been reported for state estimation of linear time-invariant (LTI) systems via wireless sensor networks. For example, the works [13] and [14] focus on delay issues in a sensor network with no dropouts, whereas [15] studies the effect of dropouts within an architecture with only one sensor node, but where additional relay nodes are allowed to process data. The paper [16] examines various information fusion strategies for distributed state estimation in sensor networks having a star topology. In the recent work [17], the authors examine sensor scheduling for networks with a tree topology and no dropouts. The issue of Kalman filter stability (i.e., boundedness of the estimation error covariance matrix) has received significant attention. In particular, [18] focused on LTI plants and a single-link architecture where dropout processes are independent and identically distributed (i.i.d.). By using a fixed-point argument, [18] established that there exists a critical dropout probability value which separates situations where the expected value of the estimator covariance matrix remains bounded from instances where it diverges, see also [19], [20] and [21]. ${ }^{1}$ The latter work examines a state estimation architecture with two channels affected by i.i.d. dropouts. The case where dropouts are described by a timehomogeneous two-state Markov chain was investigated in [23], [26]-[29]. Recently, [22] studied the one-sensor case with dropouts governed by a, more general, semi-Markov chain. Inter-alia, that work combined results of [30] with bounds established in [31] to show that, under mild conditions, the empirical covariance of the estimation error converges to a unique stationary distribution.

In the present work, we study centralized state estimation for linear time-varying systems via wireless sensor networks with a tree topology. Communication links are subject to random and possibly correlated packet dropouts. Based on motivating case studies from process industry, we assume that in-network processing is much faster than the dynamics of the system whose state is being estimated and, thus, neglect delays introduced by the network. A key contribution is the proposal of a sensor network fading model, which allows for spatial and temporal correlations of channel gains and, thereby, packet dropouts. For that purpose, we introduce a network state process. The latter models shadow fading

\footnotetext{
${ }^{1}$ If, instead of the expected value of the covariance matrix, other criteria are used, then different critical dropout probabilities will be obtained [22]-[24]. Alternatively, the works [20], [22], [25], [26] directly examine the distribution of the covariance matrix.
} 


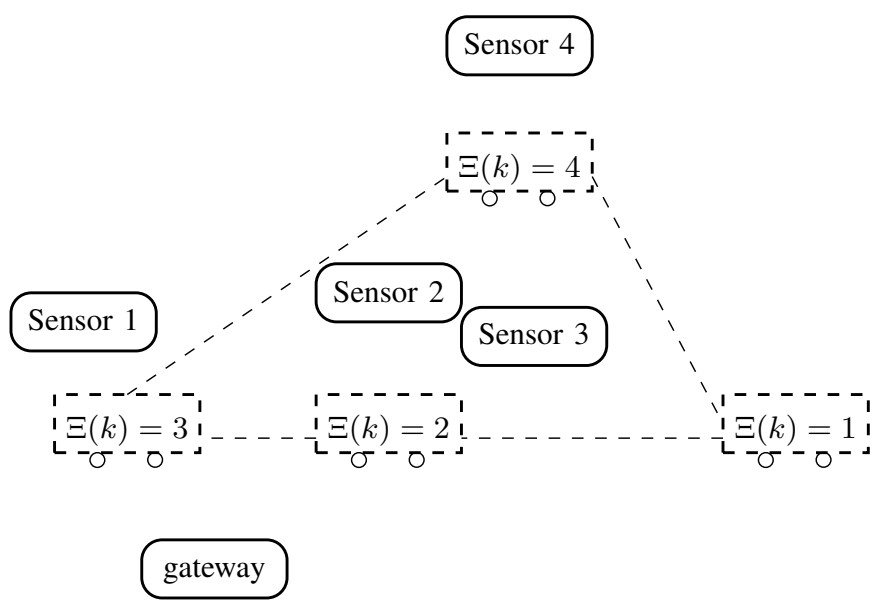

Fig. 1. Sensor network with moving robot.

effects by describing a finite set of configurations of the radio environment. The network state characterizes the channel gain distributions of the different links, which depend upon shadow and multi-path fading. To model temporal correlations of channel gains, we allow the network state process to form a (semi-)Markov chain. Our radio model structure generalizes models previously reported in the literature; see, e.g., [10], [23], [28], [32], [33] and also accounts for power and bit-rate control of sensor radio amplifiers.

As a motivating example, Fig. 1 schematizes an industrial situation, where a mobile robot is moving between four different operational points. The sensor network is set up for state estimation at the gateway. This situation can be modeled by assigning each robot position to a network state value $\Xi(k) \in\{1,2,3,4\}$. Clearly, different positions will lead to different fading environments, i.e., fading distributions between sensors and gateway.

By using elements of stochastic stability theory, we derive sufficient conditions on the system matrix and network parameters for the trace of the estimator covariance matrix to be exponentially bounded. For cases where the network state process forms a Markov Chain, the result obtained depends upon the transition probabilities of the network state and the conditional probabilities of the associated instantaneous system observation matrix to have full column rank. For network state processes described by a semi-Markov Chain, our result depends upon the transition probabilities of the embedded Markov Chain, conditional holding time distributions, and the conditional probability of a suitably defined multi-step observability matrix to have full rank. In special cases, the results obtained correspond to conditions which have been previously documented in the literature on state estimation with packet dropouts. The present paper expands upon our recent conference contribution [1] by considering a semiMarkov model for the network state.

The remainder of the paper is organized as follows: Section II describes the sensor network architecture. The proposed network fading model is presented in Section III. Section IV characterizes the associated state estimator. In Section V, we establish sufficient conditions for exponential boundedness when the network state process is Markovian. Section VI studies the special case where the network has only one sensor. Stability results for state estimation when the network states are described by a semi-Markov chain are derived in Section VII. Section VIII draws conclusions. Technical proofs are included in appendices.

Notation: We write $\mathbb{N}$ for $\{1,2, \ldots\}$, and $\mathbb{N}_{0}$ for $\mathbb{N} \cup$ $\{0\} ; \mathbb{R}$ are the real numbers, $\mathbb{R}_{\geq 0} \triangleq[0, \infty)$. The notation $\{\nu\}_{\mathbb{N}_{0}}$ refers to the sequence $\{\nu(0), \nu(1), \ldots\}$, and $\{\nu\}_{\ell}^{k}$ to $\{\nu(\ell), \nu(\ell+1), \ldots, \nu(k)\}$, with $\{\nu\}_{\ell}^{k}=\emptyset$, the empty set, whenever $\ell>k$. The notation $|\cdot|$ refers to cardinality of a set. Given any vector $v$, its Euclidean norm is denoted $\|v\|=\sqrt{v^{T} v}$, where the superscript $T$ refers to transposition. The trace of a matrix $A$ is denoted by $\operatorname{tr} A$, and its spectral norm by $\|A\| \triangleq \sqrt{\max \operatorname{eigs}\left(A^{T} A\right)}$, where $\operatorname{eigs}\left(A^{T} A\right)$ are the eigenvalues of $A^{T} A$. If a matrix $A$ is positive definite (non-negative definite), then we write $A \succ 0$ ( $A \succeq 0) ; I_{n}$ denotes the $n \times n$ identity matrix. To denote the conditional probability of an event $\Omega$ given $\Delta$, we write $\operatorname{Pr}\{\Omega \mid \Delta\}$. The expected value of a random variable $\mu$ given $\Delta$, is denoted via $\mathbf{E}\{\mu \mid \Delta\}$, whereas for the unconditional expectation we write $\mathbf{E}\{\mu\}$. A real Gaussian random variable $\mu$, with mean $\nu$ and covariance matrix $\Gamma$ is denoted by $\mu \sim \mathcal{N}(\nu, \Gamma)$.

\section{Sensor Network Architecture}

We consider uncontrolled linear time-varying $n$-dimensional systems of the form:

$$
x(k+1)=A(k) x(k)+w(k), \quad k \in \mathbb{N}_{0},
$$

where $x(0) \sim \mathcal{N}\left(x_{0}, P_{0}\right)$, with $x_{0}^{T} x_{0} \in \mathbb{R}_{\geq 0},\left\|P_{0}\right\| \in \mathbb{R}_{\geq 0}$. The driving noise process $\{w\}_{\mathbb{N}_{0}}$ is independent with $w(k) \sim$ $\mathcal{N}(0, Q(k))$, for all $k \in \mathbb{N}_{0}$.

To estimate the system state sequence $\{x\}_{\mathbb{N}_{0}}$, a collection of $M$ wireless sensors $\left\{S_{1}, \ldots, S_{M}\right\}$ is used. Each sensor provides a noisy measurement sequence $\left\{y_{m}\right\}_{\mathbb{N}_{0}}$ of the form

$$
y_{m}(k)=C_{m} x(k)+v_{m}(k), \quad m \in\{1, \ldots, M\},
$$

with $C_{m} \in \mathbb{R}^{l_{m} \times n}, l_{m} \in \mathbb{N}$. In (2), the measurement noise processes $\left\{v_{m}\right\}_{\mathbb{N}_{0}}$ are independent, with each $v_{m}(k) \sim$ $\mathcal{N}\left(0, R_{m}(k)\right){ }^{2}$ Throughout this work, we assume that $\{A\}_{\mathbb{N}_{0}}$, $\{Q\}_{\mathbb{N}_{0}}$ and $\left\{R_{m}\right\}_{\mathbb{N}_{0}}, m \in\{1, \ldots, M\}$ are deterministic and bounded sequences, known at the gateway.

The $M$ (possibly vector) measurements in (2) are to be transmitted via wireless links to a single gateway (or fusion centre), denoted $S_{0}$. Since the links are wireless, and independent on whether the medium access protocol adopted gives deterministic or random access (or a combination thereof as in the widely used IEEE 802.15.4 communication standard, see [36, Ch.4]), some measurements will be dropped by the network. The received measurement values are used to remotely estimate the state of the system (1). The present work seeks to gain understanding on the impact of measurement dropouts on estimation performance. For that purpose, we will

\footnotetext{
${ }^{2}$ In addition to measurement noise, $v_{m}(k)$ may also describe quantization effects, which we model as Gaussian and introducing possibly time-varying distortion, see also [11], [34], [35].
} 


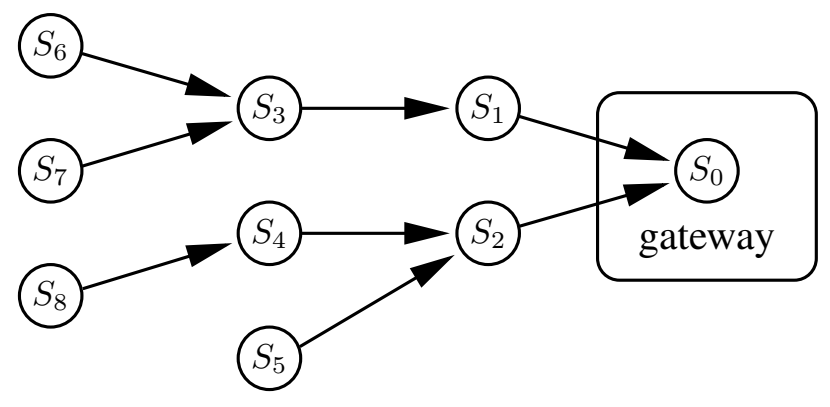

Fig. 2. Sensor network tree with 9 nodes and 8 edges.

focus on a sensor network architecture where all nodes in the network, apart from the gateway, are sensing nodes. ${ }^{3}$

As in other works, e.g., [17], we will assume that the network is much faster than the process (1) and will therefore neglect any delays experienced by the data when traveling through the network. Each sensor node aggregates its own current measurement to the received packets from incoming nodes and transmits the resulting packet to a single destination node. This reduces the energy used for listening. Sensor nodes do not buffer old data. Thus, the measurements received by the gateway at time $k$ are a subset of $\left\{y_{1}(k), y_{2}(k), \ldots, y_{M}(k)\right\}$.

It is convenient to describe the network by means of a directed graph, with vertices $\left\{S_{0}, \ldots, S_{M}\right\}$, and edges associated with the wireless links. Each sensor $S_{m}$ transmits to a single node, called its parent and henceforth denoted via $\operatorname{Par}\left(S_{m}\right)$. Thus, the graph constitutes a directed tree graph with root $S_{0}$, see also [17]. Each sensor node $S_{m}$ has a single outgoing edge, say,

$$
\mathcal{E}_{m}=\left(S_{m}, \operatorname{Par}\left(S_{m}\right)\right), \quad m \in\{1, \ldots, M\} .
$$

Furthermore, there exists a unique path from each $S_{m}$ to the gateway. We denote this path by $\operatorname{Path}\left(S_{m}\right)$, its edges by Edge $\left(\operatorname{Path}\left(S_{m}\right)\right)$ and its nodes by $\operatorname{Node}\left(\operatorname{Path}\left(S_{m}\right)\right)$.

Example 1: In the sensor network depicted in Fig. 2, the packet transmitted by $S_{3}$ at time $k \in \mathbb{N}_{0}$ contains $y_{3}(k)$ and a subset of $\left\{y_{6}(k), y_{7}(k)\right\}$. Furthermore, we have $\operatorname{Par}\left(S_{4}\right)=\operatorname{Par}\left(S_{5}\right)=S_{2}, \operatorname{Node}\left(\operatorname{Path}\left(S_{7}\right)\right)=$ $\left\{S_{7}, S_{3}, S_{1}, S_{0}\right\}$, and $\operatorname{Edge}\left(\operatorname{Path}\left(S_{7}\right)\right)=\left\{\mathcal{E}_{7}, \mathcal{E}_{3}, \mathcal{E}_{1}\right\}=$ $\left\{\left(S_{7}, S_{3}\right),\left(S_{3}, S_{1}\right),\left(S_{1}, S_{0}\right)\right\}$.

Since the links used to convey measurements from the sensors to the gateway are wireless, transmission errors are likely to occur. To study this aspect, in the following section we introduce a network model, which is physically motivated and leads to tractable analysis of the overall system

\section{Sensor Network Connectivity Model}

Channel gains are, in general, affected by path-loss, and shadow and small-scale fading; see, e.g., [7], [8], [37]. Pathloss is solely distance dependent and will therefore be constant in most industrial applications. Shadow fading is caused by large (and possibly slowly moving) objects obstructing the radio link and can therefore be correlated in time and space;

\footnotetext{
${ }^{3}$ It is worth noting, however, that relay nodes can be modeled as a sensor node with an all-zero observation matrix, see (2).
}

see also [38], [39]. If sensors are close to each other, then shadow fading may cause correlations between the individual link gains. Small-scale fading is due to local scattering in multi-paths and is commonly modeled via uncorrelated channel gain distributions.

\section{A. Network Fading Model}

To model the sensor network fading channels, we will make use of $M+1$ random variables: The network state process $\{\Xi\}_{\mathbb{N}_{0}}$, and $M$ channel (power) gains $\left\{h_{m}\right\}_{\mathbb{N}_{0}}$.

The network state process serves to capture shadow fading. It is a discrete process, i.e., we have

$$
\Xi(k) \in \mathbb{B} \triangleq\{1,2, \ldots,|\mathbb{B}|\}, \quad \forall k \in \mathbb{N}_{0},
$$

where the finite state space $\mathbb{B}$ models different configurations of the overall physical environment (such as positions of mobile objects). To incorporate temporal correlations, throughout the first part of this work, we will assume that $\{\Xi\}_{\mathbb{N}_{0}}$ is a Markov chain, as stated in Assumption 1 below. In Section VII, we will extend this model by incorporating arbitrary holding times on system states.

Assumption 1: The network states $\{\Xi\}_{\mathbb{N}_{0}}$ form a discrete (time-homogeneous) Markov chain, with transition probabilities

$$
p_{i j}=\operatorname{Pr}\{\Xi(k+1)=j \mid \Xi(k)=i\}, \quad \forall i, j \in \mathbb{B}, k \in \mathbb{N}_{0},
$$

see, e.g., [40], [41].

Each of the channel gains, $\left\{h_{m}\right\}_{\mathbb{N}_{0}}, m \in\{1,2, \ldots, M\}$, corresponds to the power gain from node $S_{m}$ to its parent node, $\operatorname{Par}\left(S_{m}\right)$. We consider block-fading, which is a common information theoretic model for fading wireless channels where the channel power gains remain invariant over a block (shorter than the coherence time of the channel) and may change from block to block [9], [10]. ${ }^{4}$ Small-scale fading is incorporated into our model by allowing channel gains at different time slots and also gains of different links to be conditionally independent for a given network state. More formally, if $\mathcal{E}_{l} \neq \mathcal{E}_{m}$ or $k \neq \ell$, then the channel gain distributions are time-homogeneous and satisfy

$$
\begin{aligned}
\operatorname{Pr}\left\{h_{l}(k) \leq a_{1}, h_{m}(\ell) \leq a_{2} \mid \Xi(k)=j, \Xi(\ell)=i\right\} \\
=\operatorname{Pr}\left\{h_{l}(k) \leq a_{1} \mid \Xi(k)=j\right\} \\
\times \operatorname{Pr}\left\{h_{m}(\ell) \leq a_{2} \mid \Xi(\ell)=i\right\},
\end{aligned}
$$

for all $a_{1}, a_{2} \in \mathbb{R}_{\geq 0}$ and all $i, j \in \mathbb{B}$. Note that in (5) we do not limit our attention to particular fading distributions. For example, our model could use Rayleigh, Rician or Nakagami distributions [7]. Furthermore, individual links are allowed to switch between different distribution classes. It is important to emphasize that, given (5), the network state process serves to describe expected channel gains. Our framework allows for spatial correlations between channel gains of individual links. It also incorporates temporal correlations, as per the Markov chain model of the network state process. For timevarying environments, our model will be more meaningful than

\footnotetext{
${ }^{4}$ This model is appropriate for many practical applications, and was considered, e.g., also in [42]
} 
simply taking long term averages of link gains; see also recent experimental studies documented in [4].

\section{B. Packet Loss}

We will assume that each data packet is either received perfectly or is completely lost and unavailable to the receiver; cf., [42]. Transmission effects are modeled via random packet dropouts at the individual links of the network. We, thus, introduce the binary stochastic communication success processes $\left\{\gamma_{m}\right\}_{\mathbb{N}_{0}}, m \in\{1,2, \ldots, M\}$, where

$\gamma_{m}(k)= \begin{cases}1 & \text { if at time } k \text { transmission via } \mathcal{E}_{m} \text { is successful } \\ 0 & \text { otherwise }\end{cases}$

The distributions of the processes $\left\{\gamma_{m}\right\}_{\mathbb{N}_{0}}$ are determined by channel gains, bit-rates and power levels. To be more specific, for each link $\mathcal{E}_{m}=\left(S_{m}, \operatorname{Par}\left(S_{m}\right)\right), m \in\{1, \ldots, M\}$, it holds that

$$
\begin{aligned}
& \operatorname{Pr}\left\{\gamma_{m}(k)=1 \mid h_{m}(k)=h, u_{m}(k)=u, b_{m}(k)=b\right\} \\
& \quad=f_{m}(h u, b),
\end{aligned}
$$

where $u_{m}(k)$ denotes the power used by the radio power amplifier of sensor $S_{m}$, and $b_{m}(k)$ the corresponding bit-rate. In (7), $f_{m}: \mathbb{R}_{>0} \times B \rightarrow[0,1]$ where $B$ is the set of allowable bit-rates. The function $f_{m}$ is monotonically increasing in the first argument (the received signal power) and monotonically decreasing in the second argument (the bit-rate). Its specific form depends on the modulation used by each node $S_{m}$, see, e.g., [8], [11]. . $^{5}$

In view of (7), power and bit-rate control can be used to counteract fading effects; see [11], [12], [43]. Throughout this work we allow transmission power levels and bit-rates to depend upon the channel gains and the network state. We, thus, introduce the following standing assumption:

Assumption 2: Power and bit-rate control laws are of the form

$$
\begin{gathered}
u_{m}(k)=\kappa_{m}\left(\Xi(k), h_{1}(k), \ldots, h_{M}(k)\right), \\
b_{m}(k)=\eta_{m}\left(\Xi(k), h_{1}(k), \ldots, h_{M}(k)\right),
\end{gathered}
$$

where $\kappa_{m}$ and $\eta_{m}, m \in\{1,2, \ldots, M\}$, are non-linear mappings.

Particular cases of (8) include the use of fixed power levels and bit-rates, fixed gain controllers with saturated outputs, $u_{m}(k)=\operatorname{sat}\left(\mathcal{K}_{m} / h_{m}(k)\right)$; and also the various power allocation policies studied in [10].

A key feature of the network model presented above and the power and bit-rate controller class considered is that, when conditioned upon the network state $\Xi$, the link transmission success processes are independent in time and of each other. For further reference, we will denote the associated success probabilities via

$$
\phi_{m \mid j} \triangleq \operatorname{Pr}\left\{\gamma_{m}(k)=1 \mid \Xi(k)=j\right\}, m \in\{1, \ldots, M\}, j \in \mathbb{B}
$$

\footnotetext{
${ }^{5}$ Fast retransmissions of individual links can be included into our framework by simply replacing $f_{m}(h u, b)$ with $1-\left(1-f_{m}(h u, b)\right)^{L}$, where $L$ is the maximum number of retransmissions allowed by the protocol and delay constraint.
}

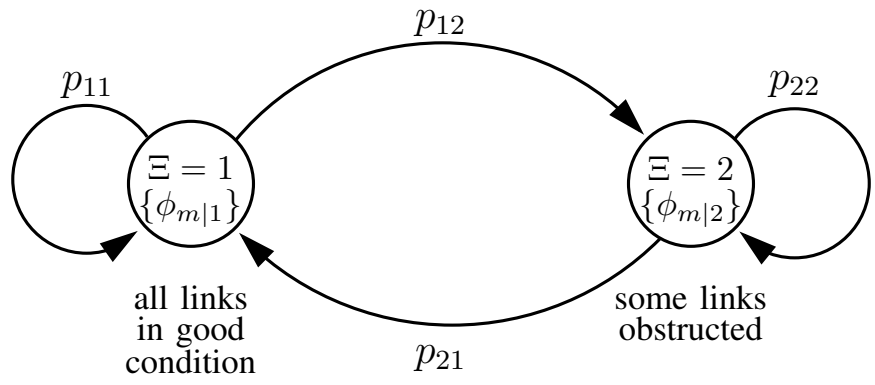

Fig. 3. Sensor network connectivity model with two states, correlated as per Assumption 1; see Example 2.

and note that for power and bit-rate control laws (8), we have

$$
\begin{array}{r}
\phi_{m \mid j}=\mathbf{E}\left\{f _ { m } \left(h_{m} \cdot \kappa_{m}\left(\Xi, h_{1}, \ldots, h_{M}\right),\right.\right. \\
\left.\left.\eta_{m}\left(\Xi, h_{1}, \ldots, h_{M}\right)\right) \mid \Xi=j\right\} .
\end{array}
$$

Thus, for given control and bit-rate policies, calculating $\phi_{m \mid j}$ involves simply taking expectation with respect to the conditional distribution of the channel gains given the network state $\Xi=j$, see (5).

Example 2: Suppose that there are only two network states: In state $\Xi=1$, all $M$ links are in good condition (with low dropout probabilities). When $\Xi(k)=2$, some of the links are obstructed by a large object, thus, having very small expected channel gains. Transition between the two network states is random and obeys the Markov chain model (4), see Fig. 3. $\square$

It is important to emphasize that the network state determines the distribution of the $M$ channel gains and thereby the distribution of the link success probabilities in (9). Despite Assumption 1, we do not require that the channel gains $\left\{h_{m}\right\}_{\mathbb{N}_{0}}$ or the dropout processes $\left\{\gamma_{m}\right\}_{\mathbb{N}_{0}}$ be Markovian. Our model encompasses, as special cases, i.i.d. transmissions [18], the Gilbert-Elliot Model [32], [44], Markovian models for the dropout processes [23], [28], [33], and channel gains described by a finite Markov chain [10]. In the one-link case, and provided Assumption 1 holds, the model presented is mathematically equivalent to the hidden Markov chain model of [45]. A key difference to [45], however, is that the present model is physically motivated and allows one to incorporate the effect of power and bit-rate control in an straightforward manner. In Section VI we will further examine these relationships.

Remark 1: The design of power and bit-rate control laws, and also how to estimate network states from dropout observations, lies outside the scope of the present work. References on the power and bit-rate control problem for state estimation with wireless links include [11], [12]. To estimate network states, one can adapt hidden Markov chain estimation techniques, as described, e.g., in [46], [47] and used in [4].

\section{State Estimation over a Sensor Network Tree WITH PACKET DROP-OUTS}

The purpose of the sensor network architecture considered is to estimate the state of the system (1) centrally at the gateway by using the measurements received from the sensors $\left\{S_{1}, S_{2}, \ldots, S_{M}\right\}$, see Fig. 2. As we have seen in Section III, 
fading channels will introduce random packet loss. From an estimation point of view, it is convenient to introduce the binary sensor-to-gateway connectivity processes $\left\{\theta_{m}\right\}_{\mathbb{N}_{0}}, m \in$ $\{1, \ldots, M\}$, where

$$
\theta_{m}(k)=\left\{\begin{array}{cc}
1 \quad & \text { if at time } k \text { transmission via } \\
& \operatorname{Path}\left(S_{m}\right) \text { is successful } \\
0 & \text { otherwise }
\end{array}\right.
$$

Remark 2: Since we assume that the network does not introduce any delays, we have

$$
\theta_{m}(k)=\prod_{\mathcal{E}_{i} \in \operatorname{Edge}\left(\operatorname{Path}\left(S_{m}\right)\right)} \gamma_{i}(k), \quad \forall m \in\{1, \ldots, M\} .
$$

Furthermore, the conditional distributions of $\left\{\theta_{m}\right\}$ given the network states can be written in terms of the individual link functions $\phi_{i \mid j}$ introduced in (9) as follows:

$$
\begin{aligned}
\operatorname{Pr} & \left\{\theta_{m}(k)=1 \mid \Xi(k)=j\right\} \\
& =\prod_{\mathcal{E}_{i} \in \operatorname{Edge}\left(\operatorname{Path}\left(S_{m}\right)\right)} \operatorname{Pr}\left\{\gamma_{i}(k)=1 \mid \Xi(k)=j\right\} \\
& =\prod_{\mathcal{E}_{i} \in \operatorname{Edge}\left(\operatorname{Path}\left(S_{m}\right)\right)} \phi_{i \mid j} .
\end{aligned}
$$

Note, however, that if $S_{m} \in \operatorname{Node}\left(\operatorname{Path}\left(S_{l}\right)\right)$, with $m \neq l$, then, in general,

$$
\begin{aligned}
& \operatorname{Pr}\left\{\theta_{m}(k)=1, \theta_{l}(k)=1 \mid \Xi(k)=j\right\} \\
& \quad \neq \operatorname{Pr}\left\{\theta_{m}(k)=1 \mid \Xi(k)=j\right\} \times \operatorname{Pr}\left\{\theta_{l}(k)=1 \mid \Xi(k)=j\right\},
\end{aligned}
$$

despite the fact that channel gain distributions satisfy (5).

We will assume that the packets transmitted from the sensors to the gateway incorporate error detection coding, see, e.g., [8], and that the gateway knows, whether received packets are error-free or not. Thus, the information available for state estimation at the gateway at time $k$ is given by

$$
\mathcal{I}(k)=\left\{\left\{\theta_{1}\right\}_{0}^{k}, \ldots,\left\{\theta_{M}\right\}_{0}^{k},\{y\}_{0}^{k}\right\},
$$

where

$$
y(k) \triangleq\left[\begin{array}{c}
\theta_{1}(k) y_{1}(k) \\
\theta_{2}(k) y_{2}(k) \\
\vdots \\
\theta_{M}(k) y_{M}(k)
\end{array}\right], \quad k \in \mathbb{N}_{0}
$$

With power and bit-rate control laws of the form (8) and given the network fading model adopted, the dropout realizations in (13) do not convey information about the system state $\{x\}_{\mathbb{N}_{0}}$. Since we have assumed that the network does not introduce any delays, it turns out that state estimation in the wireless sensor network configuration studied amounts to sampling the system (1) using the time-varying (stochastic) observation matrix

$$
C(k) \triangleq\left[\begin{array}{c}
\theta_{1}(k) C_{1} \\
\theta_{2}(k) C_{2} \\
\vdots \\
\theta_{M}(k) C_{M}
\end{array}\right], \quad k \in \mathbb{N}_{0} .
$$

Consequently, the conditional distribution of $x(k)$ given $\mathcal{I}(k-$ 1 ) is Gaussian. The conditional mean of $x(k)$,

$$
\hat{x}(k \mid k-1) \triangleq \mathbf{E}\{x(k) \mid \mathcal{I}(k-1)\}
$$

and the associated estimator covariance matrix,

$$
P(k \mid k-1) \triangleq \mathbf{E}\left\{\epsilon(k) \epsilon(k)^{T}\right\}
$$

with

$$
\epsilon(k) \triangleq x(k)-\hat{x}(k \mid k-1),
$$

satisfy the Kalman filter recursions (see, e.g., [48]):

$$
\begin{aligned}
\hat{x}(k+1 \mid k)= & A(k) \hat{x}(k \mid k-1) \\
& +K(k)(y(k)-C(k) \hat{x}(k \mid k-1)) \\
P(k+1 \mid k)=A & (k) P(k \mid k-1) A(k)^{T}+Q(k) \\
& -K(k) C(k) P(k \mid k-1) A(k)^{T}
\end{aligned}
$$

where

$$
\begin{aligned}
R(k) \triangleq & \operatorname{diag}\left(R_{1}(k), R_{2}(k), \ldots, R_{M}(k)\right), \\
K(k) \triangleq & A(k) P(k \mid k-1) C(k)^{T} \\
& \cdot\left(C(k) P(k \mid k-1) C(k)^{T}+R(k)\right)^{-1},
\end{aligned}
$$

and with initial values $P(0 \mid-1)=P_{0}$ and $\hat{x}(0 \mid-1)=x_{0}$.

It follows directly from (14) that $C(k)$ takes one of $2^{M}$ possible values. The probability distribution of $C(k)$ depends upon the current channel gains, the bit-rates and the power levels used; see (10) and (12). Thus, $\{C\}_{\mathbb{N}_{0}}$ is a random process, the recursion (17) is stochastic and the error covariance process $\{P(k+1 \mid k)\}_{k \in \mathbb{N}_{0}}$ is stochastic.

A key difference of our approach when compared to that in [13], [14], is that we consider packet dropouts. Thus, in case of open loop unstable systems (1), the estimator covariance matrix will, in general not be stationary. The situation is akin to that encountered in the context of state estimation over lossy communication links with constant dropout probabilities; see, e.g., [49], or also where dropout processes are (semi)Markovian; see, e.g., [22], [23], [28], [29], [33]. In the case under study in the present work, the plant model and transmission success probabilities are time varying, and channel gains are correlated between each other. Thus, the results of the above articles cannot be applied directly. In the following section, we will take a closer look at stochastic stability of the Kalman filter (17).

\section{Stability Analysis fOr Markovian Network STATES}

If the system (1) is unstable, then due to packet dropouts, the covariance matrix process $\{P(k+1 \mid k)\}_{k \in \mathbb{N}_{0}}$ in (17) will, in general, not converge to a fixed value and may, at times, diverge, thereby indicating poor performance of the Kalman filter. As shown in [49], this type of behaviour occurs even in the simplest scenario, where only one sensor is used and dropout probabilities are i.i.d. We will next study stability of the Kalman filter for the sensor network model presented in Section III. Towards that goal we adopt a stochastic stability notion, which captures the fact that, with dropouts, the best one can hope for is that the estimator covariance matrix be 
bounded. More precisely, we will focus on the trace of the covariance matrix, which by (15) quantifies the mean square of the estimation error, $\operatorname{tr} P(k \mid k-1)=\mathbf{E}\left\{\|\epsilon(k)\|^{2}\right\}$, and adopt the following definition, adapted from [50]:

Definition 1: The Kalman filter in (17) is said to be exponentially bounded, if there exist finite constants $\alpha$ and $\beta$ and $\rho \in[0,1)$ such that:

$$
\mathbf{E}\{\operatorname{tr} P(k \mid k-1)\} \leq \alpha \rho^{k}+\beta, \quad \forall k \in \mathbb{N}_{0} .
$$

Remark 3: Since $P(k \mid k-1) \succeq 0$, it directly follows that $\operatorname{tr} P(k \mid k-1) \geq\|P(k \mid k-1)\|=\sqrt{\max \operatorname{eigs}(P(k \mid k-1))}$ for all $k \in \mathbb{N}_{0}$. Thus, exponential boundedness of the Kalman filter implies boundedness of $\mathbf{E}\{P(k \mid k-1)\}$ as studied, for example, in [18], [19], [21] and also covariance stability, i.e., $\mathbf{E}\{\|P(k+1 \mid k)\|\}<\infty$, for all $k \in \mathbb{N}_{0}$, see, e.g., [23], [28]. Exponential boundedness has also been used in [51] for the analysis of a class of networked control systems with Markovian packet dropouts and non-vanishing disturbances. $\square$

Our analysis makes use of the process $\{r\}_{\mathbb{N}_{0}}$, where

$$
r(k) \triangleq \begin{cases}1 & \text { if } C(k) \text { is full rank } \\ 0 & \text { otherwise }\end{cases}
$$

We also introduce

$$
\nu_{i} \triangleq \operatorname{Pr}\{r(k)=0 \mid \Xi(k-1)=i\}, \quad i \in \mathbb{B},
$$

which denotes the probability of $C(k)$ not being full rank, given $\Xi(k-1)=i$. Note that, by the law of total probabilities, we have

$$
\begin{aligned}
\nu_{i}= & \sum_{j \in \mathbb{B}} \operatorname{Pr}\{r(k)=0 \mid \Xi(k-1)=i, \Xi(k)=j\} \\
& \times \operatorname{Pr}\{\Xi(k)=j \mid \Xi(k-1)=i\} \\
= & \sum_{j \in \mathbb{B}} p_{i j} \operatorname{Pr}\{r(k)=0 \mid \Xi(k)=j\}, \quad \forall i \in \mathbb{B} .
\end{aligned}
$$

Clearly, $r(k)$ is a (Boolean) function of the individual link success outcomes $\gamma_{m}(k), m \in\{1, \ldots, M\}$ and thereby depends upon the channel gains, and bit-rate and power control laws, see (10). By the discussion in Section III, it is easy to see that (provided Assumption 2 holds), $r(k)$ is temporarily independent, when conditioned upon the network state $\Xi(k)$. Furthermore, and as with the sensor-to-gateway connectivity processes $\theta_{m}(k)$, the conditional distribution $\operatorname{Pr}\{r(k) \mid \Xi(k)\}$ can be written in terms of the functions $\phi_{m \mid j}$ introduced in (9), see also Example 3 included at the end of this section.

The following theorem gives a sufficient condition for stability of the Kalman filter used for state estimation over a sensor network with Markovian channel states.

Theorem 1: Suppose that Assumptions 1 and 2 hold. If there exists $\rho \in[0,1)$ such that

$$
\max _{(i, k) \in \mathbb{B} \times \mathbb{N}_{0}} \nu_{i}\|A(k)\|^{2} \leq \rho,
$$

then the Kalman filter with the channel gain and power and bit-rate control model described in Section III is exponentially bounded.

Proof: See Appendix A.

Our result establishes a sufficient condition for exponential boundedness of the estimator covariance matrix when the

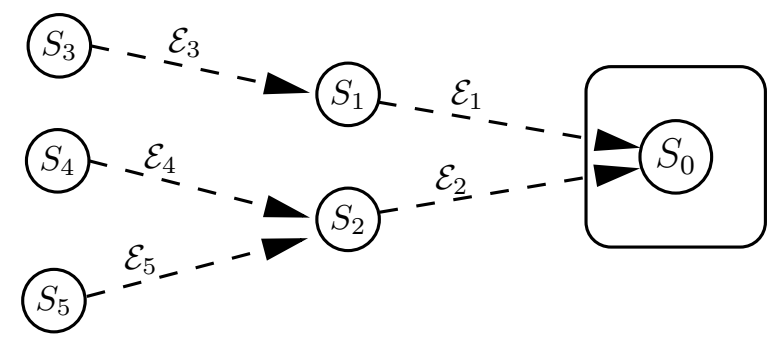

Fig. 4. Sensor network tree with $M=6$ nodes used in Example 3. Sensors $S_{3}, S_{4}$ and $S_{5}$ transmit their own measurements. At each time $k, S_{1}$ transmits $y_{1}(k)$ and, if received, also $y_{3}(k)$. Likewise, $S_{2}$ transmits $y_{2}(k)$ and a subset of $\left\{y_{4}(k), y_{5}(k)\right\}$, depending upon the transmission outcomes of links $\mathcal{E}_{4}$ and $\mathcal{E}_{5}$.

channel gains of the $M$ links are governed by the radio environment model described in Section III. The condition requires that $C(k)$ be full rank if all measurements are received and is stated in terms of a bound which involves the spectral norm of the system matrices $A(k)$, the transition probabilities of the channel state $\Xi$, and the conditional probabilities of $\{r\}_{\mathbb{N}_{0}}$. The latter are determined by the individual conditional transmission success probabilities $\phi_{m \mid j}$, and can therefore be influenced by designing the power and bit-rate control policies, see (10). The situation investigated in the present work generalizes that studied for the simpler case of having independent channel gains in our recent paper [12].

Before turning our attention to a particular case, namely when the network has only one sensor, we will first give an example which illustrates how to evaluate $\operatorname{Pr}\{r(k) \mid \Xi(k)\}$.

Example 3: Consider the subgraph of the sensor network topology of Fig. 2 having vertices $\left\{S_{0}, S_{1}, \ldots, S_{5}\right\}$, see Fig. 4 . Suppose that for $C(k)$ to be full rank (at least) three of the measurements $\left\{y_{1}(k), y_{2}(k), \ldots, y_{5}(k)\right\}$ need to be received at the gateway. Then $r(k)=1$ if and only if

$$
\left[\begin{array}{llll}
\gamma_{1}(k) & \gamma_{2}(k) & \ldots & \gamma_{5}(k)
\end{array}\right]^{T} \in \mathbb{J},
$$

where

$$
\mathbb{J} \triangleq\left\{\left[\begin{array}{l}
0 \\
1 \\
0 \\
1 \\
1
\end{array}\right],\left[\begin{array}{l}
0 \\
1 \\
1 \\
1 \\
1
\end{array}\right],\left[\begin{array}{l}
1 \\
1 \\
0 \\
0 \\
1
\end{array}\right],\left[\begin{array}{l}
1 \\
1 \\
0 \\
1 \\
0
\end{array}\right],\left[\begin{array}{l}
1 \\
1 \\
0 \\
1 \\
1
\end{array}\right],\left[\begin{array}{l}
1 \\
1 \\
1 \\
0 \\
0
\end{array}\right],\left[\begin{array}{l}
1 \\
1 \\
1 \\
0 \\
1
\end{array}\right],\left[\begin{array}{l}
1 \\
1 \\
1 \\
1 \\
0
\end{array}\right],\left[\begin{array}{l}
1 \\
1 \\
1 \\
1 \\
1
\end{array}\right]\right\} .
$$

If Assumption 2 holds, then, as noted in Section III, the link transmission success processes $\gamma_{m}$ are conditionally independent. Thus, the conditional probabilities of $C$ being full rank can be obtained from $\mathbb{J}$ as follows:

$$
\begin{aligned}
& \operatorname{Pr}\{r=1 \mid \Xi=j\}=\left(1-\phi_{1 \mid j}\right) \phi_{2 \mid j}\left(1-\phi_{3 \mid j}\right) \phi_{4 \mid j} \phi_{5 \mid j} \\
& +\left(1-\phi_{1 \mid j}\right) \phi_{2 \mid j} \phi_{3 \mid j} \phi_{4 \mid j} \phi_{5 \mid j}+\phi_{1 \mid j} \phi_{2 \mid j}\left(1-\phi_{3 \mid j}\right)\left(1-\phi_{4 \mid j}\right) \phi_{5 \mid j} \\
& +\phi_{1 \mid j} \phi_{2 \mid j}\left(1-\phi_{3 \mid j}\right) \phi_{4 \mid j}\left(1-\phi_{5 \mid j}\right)+\phi_{1 \mid j} \phi_{2 \mid j}\left(1-\phi_{3 \mid j}\right) \phi_{4 \mid j} \phi_{5 \mid j} \\
& +\phi_{1 \mid j} \phi_{2 \mid j} \phi_{3 \mid j}\left(1-\phi_{4 \mid j}\right)\left(1-\phi_{5 \mid j}\right)+\phi_{1 \mid j} \phi_{2 \mid j} \phi_{3 \mid j}\left(1-\phi_{4 \mid j}\right) \phi_{5 \mid j} \\
& +\phi_{1 \mid j} \phi_{2 \mid j} \phi_{3 \mid j} \phi_{4 \mid j}\left(1-\phi_{5 \mid j}\right)+\phi_{1 \mid j} \phi_{2 \mid j} \phi_{3 \mid j} \phi_{4 \mid j} \phi_{5 \mid j},
\end{aligned}
$$

for all $j \in \mathbb{B}$. 


\section{ThE ONE-SENSOR CASE FOR LTI SyStEMS}

Here, we focus on a particular instance of the sensor network model of interest, namely, where there is only one sensor and one edge, and the system (1) is LTI. In this case, it is easy to see that $C(k)=\gamma_{1}(k) C_{1}$ and the estimator covariance matrix in (17) satisfies

$$
\begin{gathered}
P(k+1 \mid k)=A P(k \mid k-1) A^{T}+Q-K(k) C_{1} P(k \mid k-1) A^{T} \\
K(k)=\gamma_{1}(k) A P(k \mid k-1) C_{1}^{T}\left(C_{1} P(k \mid k-1) C_{1}^{T}+R\right)^{-1} .
\end{gathered}
$$

Theorem 1 can be directly applied to this setup, yielding the following result:

Corollary 1: Consider the model introduced in Section III with $M=1$. Suppose that Assumptions 1 and 2 hold, and that $C_{1}$ in (2) is full rank. If there exists $\rho \in[0,1)$ such that

$$
\|A\|^{2} \sum_{j \in \mathbb{B}} p_{i j}\left(1-\phi_{1 \mid j}\right) \leq \rho, \quad \forall i \in \mathbb{B},
$$

then the Kalman filter is exponentially bounded.

Proof: Immediate from Theorem 1, since $r(k)=\gamma_{1}(k)$.

Kalman filtering with a single sensor-link and Markovian packet dropouts was investigated in [23], [28], [29]. Markovian dropouts correspond to the particular case of the setup considered in Corollary 1 , namely, where $\mathbb{B}=\{1,2\}, \phi_{1 \mid 1}=1$, and $\phi_{1 \mid 2}=0$, see Fig. 3. Direct calculations give that with these parameters the sufficient condition for stochastic stability (24) reduces to

$$
\|A\|^{2} \max \left(p_{12}, p_{22}\right) \leq \rho<1
$$

thus, Corollary 1 becomes akin to Theorem 3 in [28]. In a similar manner, it can be shown that the hidden Markov model of [45] (which generalizes the Gilbert loss model [32] and a fixed-length burst loss description) can be recovered by setting $\phi_{1 \mid j} \in\{0,1\}$ for all $j \in \mathbb{B}$ in the hypotheses of Corollary 1. Vice-versa, the model considered in Corollary 1, can be stated in terms of the hidden Markov chain model of [45] by considering the aggregated state process $\left\{\left(\gamma_{1}, \Xi\right)\right\}_{\mathbb{N}_{0}}$.

Our model can be further simplified by allowing for only one network state, i.e., by setting $|\mathbb{B}|=1$ in (3). In this case, we obtain a system with i.i.d. dropouts having transmission success probabilities $\operatorname{Pr}\left\{\gamma_{1}(k)=1\right\}=\phi_{1 \mid 1}$, see (9). Whilst this situation will not often be encountered in practice, it certainly is of significant system-theoretic importance, and has been extensively studied; see, e.g., [18]-[20], [22], [24]-[26], [49]. With i.i.d. dropouts, the condition (24) becomes

$$
\operatorname{Pr}\left\{\gamma_{1}(k)=0\right\}\|A\|^{2} \leq \rho<1,
$$

thereby resembling various conditions which have been reported in the literature; see [49]. ${ }^{6}$

\footnotetext{
${ }^{6}$ The i.i.d. dropout case can also be regarded as a special instance of Corollary 1 , where $\mathbb{B}=\{1,2\}, \phi_{1 \mid 1}=1, \phi_{1 \mid 2}=0, p_{21}=p_{11}$, and $p_{12}=p_{22}$ is the dropout probability.
}

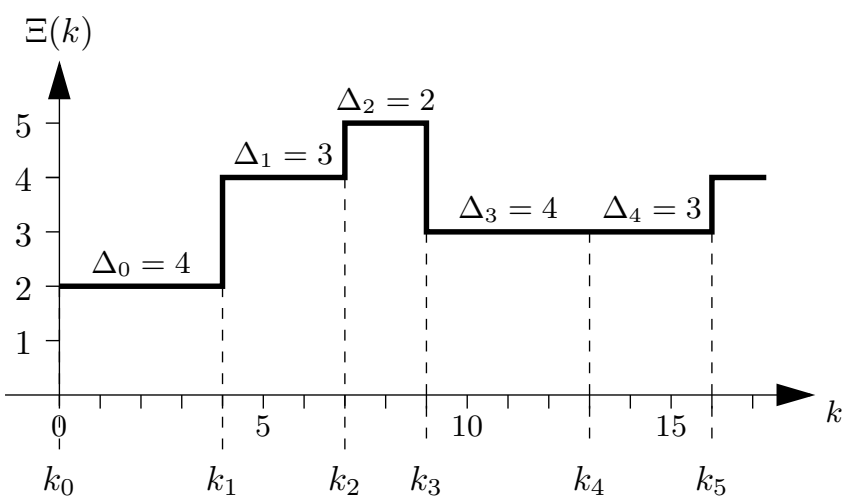

Fig. 5. Semi-Markov network state model as per Assumption 3.

\section{Network States With Arbitrary Holding TIMES}

In Sections V and VI we assumed that the network state may change at every instant $k \in \mathbb{N}_{0}$, see Assumption 1 . This model serves to describe situations where the radio environment changes relatively fast. We will next present a more general sensor network connectivity model. It allows one to impose minimum holding times on the network states and is thereby especially tailored for situations where the radio environment changes slowly.

\section{A. Semi-Markov Model}

We will allow the times between switches to follow an arbitrary probability distribution. At the instants of transitions, henceforth denoted by the ordered set

$$
\mathbb{K} \triangleq\left\{k_{\ell}\right\}_{\ell \in \mathbb{N}_{0}} \subseteq \mathbb{N}_{0}, \quad k_{0}=0,
$$

the process $\{\Xi\}_{\mathbb{K}_{0}}$ behaves like a Markov chain. Thus, our model fits into the semi-Markovian framework considered, e.g., in [22], [47], [52]-[54]. To be more precise, we introduce the following assumption:

Assumption 3: The holding times,

$$
\Delta_{\ell} \triangleq k_{\ell+1}-k_{\ell} \in \mathbb{N}, \quad \ell \in \mathbb{N}_{0}
$$

and the following transition $\Xi\left(k_{\ell+1}\right)$ are conditionally independent given (and depend only on) the current state, $\Xi\left(k_{\ell}\right)$, i.e., we have

$$
\begin{gathered}
\operatorname{Pr}\left\{\Xi\left(k_{\ell+1}\right)=j, \Delta_{\ell}=\delta \mid \Xi\left(k_{0}\right), \Xi\left(k_{1}\right) \ldots, \Xi\left(k_{\ell-1}\right),\right. \\
\left.\Xi\left(k_{\ell}\right)=i, k_{0}, \ldots, k_{\ell}\right\}=q_{i j} \psi_{i}(\delta),
\end{gathered}
$$

for all $i, j \in \mathbb{B}, \delta \in \mathbb{N}$, where

$$
\begin{aligned}
\psi_{i}(\delta) & \triangleq \operatorname{Pr}\left\{\Delta_{\ell}=\delta \mid \Xi\left(k_{\ell}\right)=i\right\} \\
q_{i j} & \triangleq \operatorname{Pr}\left\{\Xi\left(k_{\ell+1}\right)=j \mid \Xi\left(k_{\ell}\right)=i\right\},
\end{aligned}
$$

are the conditional distribution of the holding times and the transition probabilities of the embedded Markov chain $\left\{\Xi\left(k_{\ell}\right)\right\}_{\ell \in \mathbb{N}_{0}}$, respectively.

Note that if in (26) we have $\Delta_{\ell} \geq 2$, for some $\ell \in \mathbb{N}_{0}$, then

$$
\Xi\left(k_{\ell}\right)=\Xi\left(k_{\ell}+1\right)=\cdots=\Xi\left(k_{\ell}+\Delta_{\ell}-1\right) .
$$

Thus, the renewal process $\left\{\left(\Xi\left(k_{\ell}\right), k_{\ell}\right)\right\}_{\ell \in \mathbb{N}_{0}}$ describes the network state trajectory at all times $k \in \mathbb{N}_{0}$. It is worth 


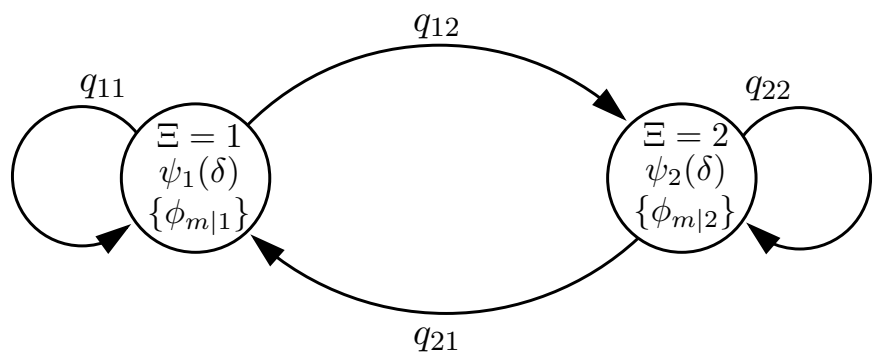

Fig. 6. Semi-Markov sensor network connectivity model as per Assumption 3 , where $\mathbb{B}=\{1,2\}$.

emphasizing that, unless $q_{i i}=0$ for all $i \in \mathbb{B}$, (27) allows for virtual transitions, i.e., where $\Xi\left(k_{\ell+1}\right)=\Xi\left(k_{\ell}\right)$, see Figs. 5 and 6 . The above transition model generalizes the Markov model in Assumption 1, see also Fig. 3, by allowing one to assign holding time distributions. Inter-alia, this serves to capture situations where the environment changes slowly, in relation to the sampling frequency of the system (1), see (29).

Example 4: Consider the scheme in Fig. 1 and assume that the position $\Xi(k)=1$ is the robot "home" position (e.g., where its batteries are charged). The robot trajectory could, for example, be described by the model in Assumption 3 with transition probabilities

$$
\begin{array}{llll}
q_{11}=0.8, & q_{12}=0.1, & q_{13}=0, & q_{14}=0.1, \\
q_{21}=0.5, & q_{22}=0.3, & q_{23}=0.1, & q_{24}=0.1, \\
q_{31}=0, & q_{32}=0.2, & q_{33}=0.5, & q_{34}=0.3, \\
q_{41}=0.6, & q_{42}=0.2, & q_{43}=0.1, & q_{44}=0.1,
\end{array}
$$

and holding time distributions

$$
\begin{aligned}
& \psi_{1}(\delta)= \begin{cases}1 & \text { if } \delta=30, \\
0 & \text { if } \delta \neq 30 .\end{cases} \\
& \psi_{2}(\delta)=\psi_{3}(\delta)=1 / 8, \quad \text { if } \delta \in\{1,2, \ldots, 8\}, \\
& \psi_{4}(\delta)=1 / 6, \quad \text { if } \delta \in\{5,6, \ldots, 10\} .
\end{aligned}
$$

In the following, we will study stochastic stability of the Kalman filter (17) for sensor network setups of this type.

\section{B. Stability Analysis}

To characterize the system behaviour with the semi-Markov model of Assumption 3, it is convenient to introduce the transition matrix $\Phi(\ell, k)$, see [48], via

$$
\begin{aligned}
& \Phi(\ell, k)=A(\ell-1) A(\ell-2) \ldots A(k), \quad \ell>k \\
& \Phi(\ell, \ell)=I_{n},
\end{aligned}
$$

so that

$x(k+i)=\Phi(k+i, k) x(k)+\sum_{\ell=0}^{i-1} \Phi(k+i, k+\ell+1) w(k+\ell)$,

expression which follows directly from (1). We also denote the observability matrix of order $t \in \mathbb{N}_{0}$ with initial step $k$, via $\mathcal{O}(k, k)=C(k)$, and

$$
\mathcal{O}(k+t, k)=\left[\begin{array}{c}
C(k) \Phi(k, k) \\
C(k+1) \Phi(k+1, k) \\
\vdots \\
C(k+t) \Phi(k+t, k)
\end{array}\right], \quad t \in \mathbb{N} .
$$

Our analysis makes use of the process $\left\{\varrho\left(k_{\ell}\right)\right\}_{k_{\ell} \in \mathbb{K}}$, where

$$
\varrho\left(k_{\ell}\right) \triangleq \begin{cases}1 & \text { if } \mathcal{O}\left(k_{\ell}+\Delta_{\ell}-1, k_{\ell}\right) \text { is full rank, } \\ 0 & \text { otherwise. }\end{cases}
$$

Clearly, $\varrho\left(k_{\ell}\right)$ is a function of the individual link success outcomes $\left\{\gamma_{m}(k)\right\}_{k_{\ell}}^{k_{\ell+1}-1}, m \in\{1, \ldots, M\}$ and of $\{A(k)\}_{k_{\ell}}^{k_{\ell+1}-2}$. Akin to what was done in Section V, see (20), we define

$$
\begin{aligned}
& \mu_{i}\left(k_{\ell}, \delta\right) \triangleq \operatorname{Pr}\left\{\varrho\left(k_{\ell}\right)=0 \mid \Xi\left(k_{\ell}-1\right)=i, \Delta_{\ell}=\delta\right\} \\
& =\sum_{j \in \mathbb{B}} \operatorname{Pr}\left\{\varrho\left(k_{\ell}\right)=0 \mid \Xi\left(k_{\ell}-1\right)=i, \Delta_{\ell}=\delta, \Xi\left(k_{\ell}\right)=j\right\} \\
& \quad \times \operatorname{Pr}\left\{\Xi\left(k_{\ell}\right)=j \mid \Xi\left(k_{\ell}-1\right)=i, \Delta_{\ell}=\delta\right\} \\
& =\sum_{j \in \mathbb{B}} q_{i j} \operatorname{Pr}\left\{\varrho\left(k_{\ell}\right)=0 \mid \Xi\left(k_{\ell}\right)=j, \Delta_{\ell}=\delta\right\}, \quad i \in \mathbb{B},
\end{aligned}
$$

which denotes the probability of $\mathcal{O}\left(k_{\ell}+\delta-1, k_{\ell}\right)$ not being full rank, conditioned on $\Xi\left(k_{\ell}-1\right)=i$. The following result establishes sufficient conditions for exponential boundedness of the Kalman filter (17) for cases where $\left\{\Delta_{\ell}\right\}_{\ell \in \mathbb{N}_{0}}$ has bounded support.

Theorem 2: Suppose that Assumptions 2 and 3 hold and that there exists a finite value $\sigma$ such that $\Delta_{\ell} \leq \sigma$, for all $\ell \in \mathbb{N}_{0} \cdot{ }^{7}$ If there exists $\rho \in[0,1)$ such that

$$
\max _{\left(i, k_{\ell}\right) \in \mathbb{B} \times \mathbb{K}} \sum_{\delta=1}^{\sigma} \mu_{i}\left(k_{\ell}, \delta\right) \sum_{j \in \mathbb{B}} \psi_{j}(\delta) q_{i j}\left\|\Phi\left(k_{\ell}+\delta, k_{\ell}\right)\right\|^{2} \leq \rho^{\sigma},
$$

then the Kalman filter in (17) is exponentially bounded.

Proof: See Appendix B.

To elucidate the condition (32), it is convenient to recall the definitions (28) and (31) and note that for a given holding time $\Delta_{\ell}=\delta \in\{1,2, \ldots, \sigma\}$, we have

$$
\begin{aligned}
\mu_{i}\left(k_{\ell}, \delta\right) \sum_{j \in \mathbb{B}} & \psi_{j}(\delta) q_{i j} \\
= & \operatorname{Pr}\left\{\varrho\left(k_{\ell}\right)=0 \mid \Xi\left(k_{\ell}-1\right)=i, \Delta_{\ell}=\delta\right\} \\
& \times \sum_{j \in \mathbb{B}} \operatorname{Pr}\left\{\Delta_{\ell}=\delta \mid \Xi\left(k_{\ell}\right)=j\right\} \\
& \times \operatorname{Pr}\left\{\Xi\left(k_{\ell}\right)=j \mid \Xi\left(k_{\ell}-1\right)=i\right\} \\
= & \operatorname{Pr}\left\{\varrho\left(k_{\ell}\right)=0 \mid \Xi\left(k_{\ell}-1\right)=i, \Delta_{\ell}=\delta\right\} \\
& \times \operatorname{Pr}\left\{\Delta_{\ell}=\delta \mid \Xi\left(k_{\ell}-1\right)=i\right\} .
\end{aligned}
$$

Thus, the left-hand-side of (32) extends the left-hand-side of (22) for use in the semi-Markov model by averaging non-full-rank observation outcomes over finite horizons. Here it is worth noting that the Markovian network model of

\footnotetext{
${ }^{7}$ Since our model allows for virtual transitions, we do not impose that the
} network state changes, at most, every $\sigma$ instants. 
Assumption 1 corresponds to the special instance of the model in Assumption 3, wherein $p_{i j}=q_{i j}$ for all $i, j \in \mathbb{B}$, and $\Delta_{\ell}=1$ for all $\ell \in \mathbb{N}_{0}$, so that $\psi_{j}(1)=1$ for all $j \in \mathbb{B}, \sigma=1$ and $\mathbb{K}=\mathbb{N}_{0}$. In addition, we have $\Phi(k+1, k)=A(k)$,

$$
\begin{aligned}
\mu_{i}(k, 1) & =\sum_{j \in \mathbb{B}} p_{i j} \operatorname{Pr}\left\{\varrho(k)=0 \mid \Xi(k)=j, \Delta_{\ell}=1\right\} \\
& =\sum_{j \in \mathbb{B}} p_{i j} \operatorname{Pr}\{r(k)=0 \mid \Xi(k)=j\}=\nu_{i},
\end{aligned}
$$

and $\sum_{j \in \mathbb{B}} q_{i j}=1$ for all $i \in \mathbb{B}$. Consequently, it is easy to see that, in this case, the condition (32) condenses into (22) and we recover the result established in Theorem 1.

Remark 4: In contrast to Theorem 1, Theorem 2 does not require that the matrix $C(k)$ be full rank with non-zero probability in order to establish exponential boundedness of the Kalman filter. Inter-alia, Theorem 2 requires that when no dropouts occur the system (1)-(2) be observable over horizons of length $\sigma$.

\section{Example}

To illustrate the use of Theorem 2, we examine a simple LTI plant model with $M=1$ sensor and where

$$
A=\left[\begin{array}{cc}
1.25 & 0 \\
1 & 1.1
\end{array}\right], \quad C_{1}=\left[\begin{array}{ll}
1 & 1
\end{array}\right]
$$

taken from [18]. The connectivity of $S_{1}$ to the gateway is described by two possible configurations, $\Xi(k) \in \mathbb{B}=\{1,2\}$, which obey Assumption 3, with

$$
\begin{array}{ll}
\psi_{1}(\delta)=1 / 5, & \text { if } \delta \in\{1,2, \ldots, 5\}, \\
\psi_{2}(\delta)=1 / 7, & \text { if } \delta \in\{1,2, \ldots, 7\},
\end{array}
$$

see Fig 6. Power and bit-rate control laws are of the form (8).

For one-sensor LTI systems, we have $C(k)=\gamma_{1}(k) C_{1}$, and $\Phi(k+t, k)=A^{t}$ (which is obtained by setting $A(k)=A$ ), for all $k, t \in \mathbb{N}_{0}$, thus,

$$
\mathcal{O}\left(k_{\ell}+\delta-1, k_{\ell}\right)=\left[\begin{array}{c}
\gamma_{1}\left(k_{\ell}\right) C_{1} \\
\gamma_{1}\left(k_{\ell}+1\right) C_{1} A \\
\vdots \\
\gamma_{1}\left(k_{\ell}+\delta-1\right) C_{1} A^{\delta-1}
\end{array}\right] .
$$

It is easy to verify that for the system matrices given in (33), the matrix

$$
\left[\begin{array}{c}
C_{1} \\
C_{1} A^{r}
\end{array}\right]
$$

is invertible, for all $r \in\{1, \ldots, 6\}$. Therefore, $\varrho\left(k_{\ell}\right)=0$, if and only if

$$
\sum_{t=0}^{\Delta_{\ell}-1} \gamma_{1}\left(k_{\ell}+t\right) \leq 1
$$

and the conditional probabilities in (31) become

$$
\begin{aligned}
& \mu_{i}\left(k_{\ell}, \delta\right) \\
& =\sum_{j=1}^{2} q_{i j} \operatorname{Pr}\left\{\sum_{t=0}^{\Delta_{\ell}-1} \gamma_{1}\left(k_{\ell}+t\right)=0 \mid \Xi\left(k_{\ell}\right)=j, \Delta_{\ell}=\delta\right\} \\
& \quad+\sum_{j=1}^{2} q_{i j} \operatorname{Pr}\left\{\sum_{t=0}^{\Delta_{\ell}-1} \gamma_{1}\left(k_{\ell}+t\right)=1 \mid \Xi\left(k_{\ell}\right)=j, \Delta_{\ell}=\delta\right\} .
\end{aligned}
$$

Thus, for $\delta=1$ we obtain

$$
\begin{aligned}
\mu_{i}\left(k_{\ell}, 1\right) & =\sum_{j=1}^{2} q_{i j} \operatorname{Pr}\left\{\gamma_{1}\left(k_{\ell}\right) \in\{0,1\} \mid \Xi\left(k_{\ell}\right)=j, \Delta_{\ell}=1\right\} \\
& =\sum_{j=1}^{2} q_{i j}=1,
\end{aligned}
$$

whereas, for $\delta \geq 2$,

$$
\mu_{i}\left(k_{\ell}, \delta\right)=\sum_{j=1}^{2} q_{i j}\left(\left(1-\phi_{1 \mid j}\right)^{\delta}+\delta\left(1-\phi_{1 \mid j}\right)^{\delta-1} \phi_{1 \mid j}\right),
$$

see (9). Given the above, Theorem 2 establishes that the Kalman filter (17) is exponentially bounded if there exists $\rho \in[0,1)$, such that

$$
\begin{aligned}
& \max _{i \in\{1,2\}} \sum_{\delta=1}^{7}\left\|A^{\delta}\right\|^{2} \mu_{i}\left(k_{\ell}, \delta\right)\left(\psi_{1}(\delta) q_{i 1}+\psi_{2}(\delta) q_{i 2}\right) \\
& =\max _{i \in\{1,2\}}\left(\frac{q_{i 1}}{5}+\frac{q_{i 2}}{7}\right)\left(\|A\|^{2}\right. \\
& \left.\quad+\sum_{\delta=2}^{5}\left\|A^{\delta}\right\|^{2} \mu_{i}\left(k_{\ell}, \delta\right)\right)+\frac{q_{i 2}}{7} \sum_{\delta=6}^{7}\left\|A^{\delta}\right\|^{2} \mu_{i}\left(k_{\ell}, \delta\right) \leq \rho^{7},
\end{aligned}
$$

where $\mu_{i}\left(k_{\ell}, \delta\right)$ are given in (34).

\section{CONCLUSIONS}

In this work we have studied Kalman filtering for state estimation over a wireless sensor network. Since the radio links between the nodes are fading, even if alleviated by power and bit-rate control, packet drops may occur. To model different radio connectivity configurations of the environment, we have introduced a network state process. Through the use of stochastic stability methods, we have derived sufficient conditions for the Kalman filter covariance matrix to be exponentially bounded when the underlying network state is described by a (semi-)Markov chain. Under this assumption, channel gains will be correlated over space and time, which is a suitable model when considering shadow fading. In particular cases, the sufficient condition obtained reduce to stability results previously documented in the literature.

Future work includes complementing the sufficient conditions for exponential boundedness of the estimator presented with necessary ones, and using the results for the design of power and bit-rate control and re-routing strategies. Also of interest is extending the fading network model proposed to more general topologies and study its use for the analysis and design of closed loop networked control system architectures.

\section{REFERENCES}

[1] D. E. Quevedo, A. Ahlén, and K. H. Johansson, "Stability of state estimation over sensor networks with Markovian fading channels," in Proc. IFAC World Congr., 2011.

[2] J. Chen, K. H. Johansson, S. Olariu, I. C. Paschalidis, and Stokmenovic, "Guest editorial special issue on wireless sensor and actuator networks," IEEE Trans. Automat. Contr., vol. 56, no. 11, pp. 2244-2246, Oct. 2011.

[3] R. Alur, A. D'Innocenzo, K. H. Johansson, G. J. Pappas, and G. Weiss, "Compositional modeling and analysis of multi-hop control networks," IEEE Trans. Automat. Contr., vol. 56, no. 10, pp. 2345-2357, Oct. 2011. 
[4] M. Björkbom, S. Nethi, L. M. Eriksson, and R. Jäntti, "Wireless control system design and co-simulation," Contr. Eng. Pract., vol. 19, no. 9, pp. 1075-1086, Sept. 2011.

[5] M. Ilyas, I. Mahgoub, and L. Kelly, Handbook of Sensor Networks: Compact Wireless and Wired Sensing Systems. Boca Raton, FL, USA: CRC-Press, Inc, 2004.

[6] X. Shen, Q. Zhang, and R. Caiming Qiu, "Wireless sensor networking [guest ed.]," IEEE Wireless Commun., vol. 14, no. 6, pp. 4-5, Dec. 2007.

[7] A. Goldsmith, Wireless Communications. Cambridge University Press, 2005.

[8] J. G. Proakis, Digital Communications, 3rd ed. New York, N.Y.: McGraw-Hill, 1995.

[9] G. Caire, G. Taricco, and E. Biglieri, "Optimum power control over fading channels," IEEE Trans. Inform. Theory, vol. 45, no. 5, pp. 14681489, July 1999.

[10] R. A. Berry and R. G. Gallager, "Communication over fading channels with delay constraints," IEEE Trans. Inform. Theory, vol. 48, no. 5, pp. 1135-1149, May 2002.

[11] D. E. Quevedo, A. Ahlén, and J. Østergaard, "Energy efficient state estimation with wireless sensors through the use of predictive power control and coding," IEEE Trans. Signal Processing, vol. 58, no. 9, pp. 4811-4823, Sept. 2010

[12] D. E. Quevedo, A. Ahlén, A. S. Leong, and S. Dey, "On Kalman filtering over fading wireless channels with controlled transmission powers," Automatica, vol. 48, no. 7, pp. 1306-1316, July 2012.

[13] L. Shi, A. Capponi, K. H. Johansson, and R. M. Murray, "Resource optimization in a wireless sensor network with guaranteed estimator performance," IET Control Theory Appl., vol. 4, no. 5, pp. 710-723, 2010.

[14] L. Shi, "Kalman filtering over graphs: Theory and applications," IEEE Trans. Automat. Contr., vol. 54, no. 9, pp. 2230-2234, Sept. 2009.

[15] V. Gupta, A. F. Dana, J. P. Hespanha, R. M. Murray, and B. Hassibi, "Data transmission over networks for estimation and control," IEEE Trans. Automat. Contr., vol. 54, no. 8, pp. 1807-1819, Aug. 2009.

[16] A. Chiuso and L. Schenato, "Information fusion strategies and performance bounds in packet-drop networks," Automatica, vol. 47, pp. 1304 1316, July 2011.

[17] Y. Mo, E. Garone, A. Casavola, and B. Sinopoli, "Stochastic sensor scheduling for energy constrained estimation in multi-hop wireless sensor networks," IEEE Trans. Automat. Contr, vol. 56, no. 10, pp 2489-2495, Oct. 2011.

[18] B. Sinopoli, L. Schenato, M. Franceschetti, K. Poolla, M. I. Jordan, and S. S. Sastry, "Kalman filtering with intermittent observations," IEEE Trans. Automat. Contr., vol. 49, no. 9, pp. 1453-1464, Sept. 2004.

[19] K. Plarre and F. Bullo, "On Kalman filtering for detectable systems with intermittent observations," IEEE Trans. Automat. Contr., vol. 54, no. 2, pp. 386-390, Feb. 2009.

[20] S. Kar, B. Sinopoli, and J. M. F. Moura, "Kalman filtering with intermittent observations: Weak convergence to a stationary distribution," IEEE Trans. Automat. Contr., vol. 57, no. 2, pp. 405-420, Feb. 2012.

[21] X. Liu and A. Goldsmith, "Kalman filtering with partial observation losses," in Proc. IEEE Conf. Decis. Contr., Paradise Island, Bahamas, 2004, pp. 4180-4186.

[22] A. Censi, "Kalman filtering with intermittent observations: Convergence for semi-Markov chains and an intrinsic performance measure," IEEE Trans. Automat. Contr., vol. 56, no. 2, pp. 376-381, Feb. 2011.

[23] M. Huang and S. Dey, "Stability of Kalman filtering with Markovian packet losses," Automatica, vol. 43, no. 4, pp. 598-607, Apr. 2007.

[24] E. Rohr, D. Marelli, and M. Fu, "Statistical properties of the error covariance in a Kalman filter with random measurement losses," in Proc. IEEE Conf. Decis. Contr., Atlanta, GA USA, 2010.

[25] M. Epstein, L. Shi, A. Tiwari, and R. M. Murray, "Probabilistic performance of state estimation across a lossy network," Automatica, vol. 44, no. 12, pp. 3046-3053, Dec. 2008.

[26] L. Shi, M. Epstein, and R. M. Murray, "Kalman filtering over a packetdropping network: A probabilistic perspective," IEEE Trans. Automat. Contr., vol. 55, no. 3, pp. 594-604, March 2010.

[27] Z. Jin, V. Gupta, and R. Murray, "State estimation over packet dropping networks using multiple description coding," Automatica, vol. 42, no. 9, pp. 1441-1452, Sept. 2006.

[28] L. Xie and L. Xie, "Stability of a random Riccati equation with Markovian binary switching," IEEE Trans. Automat. Contr., vol. 53, no. 7, pp. 1759-1764, August 2008.

[29] K. You, M. Fu, and L. Xie, "Mean square stability for Kalman filtering with Markovian packet losses," Automatica, vol. 47, no. 12, pp. 26472657, Dec. 2011.
[30] Ö. Stenflo, "Iterated function systems controlled by a semi-Markov chain," Theory Stoch. Process., vol. 2, no. 18, pp. 305-313, 1996.

[31] P. Bougerol, "Kalman filtering with random coefficients and contractions," SIAM Journal on Control and Optimization, vol. 31, no. 4, pp. 942-959, July 1993.

[32] E. N. Gilbert, "Capacity of a burst-noise channel," The Bell Syst. Tech. J., vol. 39, pp. 1253-1265, Sept. 1960.

[33] S. C. Smith and P. Seiler, "Estimation with lossy measurements: Jump estimators for jump systems," IEEE Trans. Automat. Contr., vol. 48, no. 12, pp. 2163-2171, Dec. 2003.

[34] D. E. Quevedo, J. Østergaard, and D. Nešić, "Packetized predictive control of stochastic systems over bit-rate limited channels with packet loss," IEEE Trans. Automat. Contr, vol. 56, no. 12, pp. 2854-2868, Dec. 2011.

[35] P. Minero, M. Franceschetti, S. Dey, and G. N. Nair, "Data rate theorem for stabilization over time-varying feedback channels," IEEE Trans. Automat. Contr., vol. 54, no. 2, pp. 243-255, Feb. 2009.

[36] P. Park, "Modeling, analysis, and design of wireless sensor network protocols," Ph.D. dissertation, KTH Electrical Engineering, Stockholm, Sweden, 2011.

[37] A. Ghaffarkhah and Y. Mostofi, "Communication-aware motino planning in mobile networks," IEEE Trans. Automat. Contr., vol. 56, no. 10, pp. 2478-2485, Oct. 2011.

[38] M. Gudmundson, "Correlation model for shadow fading in mobile radio systems," Electron. Lett., vol. 27, no. 23, pp. 2145-2146, Nov. 1991.

[39] P. Agrawal and N. Patwari, "Correlated link shadow fading in multi-hop wireless networks," IEEE Trans. Wireless Commun., vol. 8, no. 8, pp. 4024-4036, Aug. 2009.

[40] P. Brémaud, Markov Chains. New York, N.Y.: Springer, 1999.

[41] E. Çinlar, Introduction to Stochastic Processes. Prentice Hall, Englewood Cliffs, NJ, 1975.

[42] Y. Mostofi and R. M. Murray, "To drop or not to drop: Design principles for Kalman filtering over wireless fading channels," IEEE Trans. Automat. Contr., vol. 54, no. 2, pp. 376-381, Feb. 2009.

[43] N. A. Pantazis and D. D. Vergados, "A survey on power control issues in wireless sensor networks," IEEE Commun. Surv. Tutorials, vol. 9, no. 4, pp. 86-107, 2007.

[44] E. O. Elliot, "Estimates of error rates for codes on burst-noise channels," The Bell Syst. Tech. J., vol. 42, pp. 1977-1997, Sept. 1963.

[45] A. K. Fletcher, S. Rangan, and V. K. Goyal, "Estimation from lossy sensor data: Jump linear modeling and Kalman filtering," in Proc. ACM/IEEE Int. Conf. Information Processing in Sensor Networks, 2004, pp. 251-258.

[46] L. R. Rabiner, "A tutorial on hidden Markov models and selected applications in speech recognition,", Proc. IEEE, vol. 77, no. 2, pp. 257-286, Feb. 1989.

[47] V. S. Barbu and N. Limnios, Semi-Markov Chains and Hidden SemiMarkov Models toward Applications. Springer, 2008.

[48] B. D. O. Anderson and J. Moore, Optimal Filtering. Englewood Cliffs, NJ: Prentice Hall, 1979.

[49] L. Schenato, B. Sinopoli, M. Franceschetti, K. Poolla, and S. S. Sastry, "Foundations of control and estimation over lossy networks," Proc. IEEE, vol. 95, no. 1, pp. 163-187, Jan. 2007.

[50] T.-J. Tarn and Y. Rasis, "Observers for nonlinear stochastic systems," IEEE Trans. Automat. Contr, vol. 21, no. 4, pp. 441-448, August 1976.

[51] D. E. Quevedo and D. Nešić, "Robust stability of packetized predictive control of nonlinear systems with disturbances and Markovian packet dropouts," Automatica, accepted for publication.

[52] E. Çinlar, "Markov renewal theory," Adv. Applied Prob., vol. 1, no. 2 , pp. 123-187, 1969.

[53] R. A. Howard, Dynamic Probabilistic Systems, Vol. II: Semi-Markov and Decision Processes. John Wiley \& Sons, 1971.

[54] L. Kleinrock, Queueing Systems. John Wiley \& Sons, 1976.

[55] D. S. Bernstein, Matrix Mathematics, 2nd ed. Princeton, N.J.: Princeton University Press, 2009.

[56] H. Kushner, Introduction to Stochastic Control. New York, N.Y.: Holt, Rinehart and Winston, Inc., 1971.

[57] S. P. Meyn, "Ergodic theorems for discrete time stochastic systems using a stochastic Lyapunov function," SIAM Journal on Control and Optimization, vol. 27, no. 6, pp. 1409-1439, Nov. 1989.

\section{APPENDIX}

\section{A. Proof of Theorem 1}

We first prepare two preliminary lemmas: 
Lemma 1: Suppose that Assumptions 1 and 2 hold. Then the composite process $\{Z\}_{\mathbb{N}_{0}}$ defined via

$$
Z(k) \triangleq(P(k \mid k-1), \Xi(k-1)),
$$

is a Markov chain.

Proof: Recall that the network state $\{\Xi\}_{\mathbb{N}_{0}}$ is Markovian and that, for given network states, the dropout processes are independent. Therefore, the distribution of the matrix $C(k)$ satisfies, for all $k \in \mathbb{N}_{0}$,

$\operatorname{Pr}\{C(k) \mid \Xi(k-1), \Xi(k-2), \ldots\}=\operatorname{Pr}\{C(k) \mid \Xi(k-1)\}$.

The result follows from (17), since $\{A\}_{\mathbb{N}_{0}},\{Q\}_{\mathbb{N}_{0}}$ and $\{R\}_{\mathbb{N}_{0}}$ are deterministic sequences.

Lemma 2: Suppose that Assumption 1 holds and define

$$
V_{k} \triangleq \operatorname{tr} P(k \mid k-1),
$$

see (16). Then $V_{k} \in \mathbb{R}_{\geq 0}$ for all $k \in \mathbb{N}_{0}$. Furthermore, there exists $W \in \mathbb{R}_{\geq 0}$, such that, for all $k \in \mathbb{N}_{0}$,

$\mathbf{E}\left\{V_{k+1} \mid Z(k)=(P, i)\right\} \leq W+\nu_{i}\left(\|A(k)\|^{2} \operatorname{tr} P+\operatorname{tr} Q(k)\right)$,

where $Z(k)$ is as defined in (35), and $\nu_{i}$ in (20).

Proof: The fact that $V_{k}$ is non-negative follows directly from (36). To prove (37), it is convenient to recall (19) and condition as follows:

$$
\begin{aligned}
& \mathbf{E}\left\{V_{k+1} \mid Z(k)\right\} \\
& \quad=\mathbf{E}\left\{V_{k+1} \mid Z(k), r(k)=1\right\} \operatorname{Pr}\{r(k)=1 \mid Z(k)\} \\
& \quad+\mathbf{E}\left\{V_{k+1} \mid Z(k), r(k)=0\right\} \operatorname{Pr}\{r(k)=0 \mid Z(k)\} .
\end{aligned}
$$

We next examine the cases $r(k) \in\{0,1\}$ separately.

1) For $r(k)=1, C(k)$ is full rank. Therefore, a simple predictor for $x(k+1)$ given $y(k) \subset \mathcal{I}(k)$, is given by

$$
\check{x}(k+1)=A(k)\left(C(k)^{T} C(k)\right)^{-1} C(k)^{T} y(k),
$$

in which case

$\check{x}(k+1)-x(k+1)=A(k)\left(C(k)^{T} C(k)\right)^{-1} C(k)^{T} v(k)-w(k)$, where $v(k) \triangleq\left[\begin{array}{llll}v_{1}(k)^{T} & v_{2}(k)^{T} \quad \ldots & v_{M}(k)^{T}\end{array}\right]^{T}$. Since, by assumption, $\{A\}_{\mathbb{N}_{0}},\{Q\}_{\mathbb{N}_{0}}$ and $\{R\}_{\mathbb{N}_{0}}$ are bounded, there exists a constant $W \in \mathbb{R}_{\geq 0}$, such that

$\mathbf{E}\left\{(\check{x}(k+1)-x(k+1))(\check{x}(k+1)-x(k+1))^{T}\right\} \preceq(W / n) I_{n}$.

Since the Kalman filter gives the minimum conditional error covariance matrix, and by the fact that for any square matrix $F, \mathbf{E}\{\operatorname{tr} F\}=\operatorname{tr} \mathbf{E}\{F\}$, we obtain the bound ${ }^{8}$

$$
\begin{gathered}
\mathbf{E}\left\{V_{k+1} \mid Z(k), r(k)=1\right\} \operatorname{Pr}\{r(k)=1 \mid Z(k)\} \\
\leq W \operatorname{Pr}\{r(k)=1 \mid Z(k)\} \leq W .
\end{gathered}
$$

2) For $r(k)=0$, the estimator covariance matrix $P(k+1 \mid k)$ is upper-bounded by the worst case, where $\gamma_{m}(k)=0, \forall m \in$ $\{1,2, \ldots, M\}$. We, thus have

$\mathbf{E}\left\{V_{k+1} \mid Z(k)=(P, i), r(k)=0\right\} \leq \mathbf{E}\left\{V_{k+1} \mid Z(k)=(P, i)\right.$, $\left.\gamma_{1}(k)=\gamma_{2}(k)=\ldots \gamma_{M}(k)=0\right\}=\operatorname{tr}\left(A(k) P A(k)^{T}+Q(k)\right)$ $=\operatorname{tr}\left(A(k)^{T} A(k) P\right)+\operatorname{tr} Q(k) \leq\|A(k)\|^{2} \operatorname{tr} P+\operatorname{tr} Q(k)$,

\footnotetext{
${ }^{8}$ Clearly, (39) is not a tight bound, but it suffices for our purpose.
}

where we have used (17) and [55, Fact 8.12.29].

To calculate $\operatorname{Pr}\{r(k)=0 \mid Z(k)=(P, i)\}$, we condition upon $\Xi(k)$ and use Assumption 1:

$$
\begin{aligned}
\operatorname{Pr}\{r(k)=0 \mid Z(k)=(P, i)\} \\
=\sum_{j \in \mathbb{B}} \operatorname{Pr}\{r(k)=0 \mid P(k \mid k-1)=P, \Xi(k-1)=i, \Xi(k)=j\} \\
\times \operatorname{Pr}\{\Xi(k)=j \mid P(k \mid k-1)=P, \Xi(k-1)=i\} \\
=\sum_{j \in \mathbb{B}} \operatorname{Pr}\{r(k)=0 \mid \Xi(k)=j\} \\
\times \operatorname{Pr}\{\Xi(k)=j \mid \Xi(k-1)=i\}=\nu_{i} .
\end{aligned}
$$

The result follows upon replacing (39)-(41) into (38).

Proof of Theorem 1: We will use a stochastic Lyapunov function approach with candidate function $V_{k}$ introduced in (36); see, e.g., [56], [57]. By Lemma 2, we have

$$
\begin{aligned}
0 \leq \mathbf{E} & \left\{V_{k+1} \mid Z(k)=(P, i)\right\} \\
& \leq W+\left(\|A(k)\|^{2} \operatorname{tr} P+\operatorname{tr} Q(k)\right) \nu_{i} \\
& \leq \nu_{i}\|A(k)\|^{2} V_{k}+\bar{\beta} \leq \rho V_{k}+\bar{\beta}, \quad \forall k \in \mathbb{N}_{0},
\end{aligned}
$$

where $\rho \in[0,1)$ is as in (22) and

$$
\bar{\beta} \triangleq W+\max _{i \in \mathbb{N}_{0}} \nu_{i} \times \max _{k \in \mathbb{K}} \operatorname{tr} Q(k) \in \mathbb{R}_{\geq 0} .
$$

Since (42) holds for all $Z(k)=(P(k \mid k-1), \Xi(k-1))$ and, by Lemma $1,\{Z\}_{\mathbb{N}_{0}}$ is Markovian, we can use [57, Prop. 3.2] to conclude that (42) is a sufficient condition for

$$
\begin{gathered}
0 \leq \mathbf{E}\left\{V_{k} \mid Z(0)=Z\right\} \leq \rho^{k} V_{0}+\bar{\beta} \sum_{i=0}^{k-1} \rho^{i} \\
=\rho^{k} V_{0}+\bar{\beta} \frac{1-\rho^{k}}{1-\rho}, \quad \forall k \in \mathbb{N}_{0} .
\end{gathered}
$$

Therefore, upon noting that $P(0 \mid-1)=P_{0}$ is given, (43) gives that (18) holds with $\alpha=\rho V_{0}$ and $\beta=\bar{\beta} /(1-\rho)$.

\section{B. Proof of Theorem 2}

To derive our result, we will first focus on the time-instances $\mathbb{K}$ defined in (25). The key property we will use is that whilst in this case $\{\Xi\}_{\mathbb{N}_{0}}$ is not Markovian, the embedded chain $\{\Xi\}_{\mathbb{K}_{0}}$ is Markovian. We begin by extending Lemmas 1 and 2 to the model in Assumption 3.

Lemma 3: Suppose that Assumptions 2 and 3 hold. Then $\{Z\}_{\mathbb{K}_{0}}$ defined as in (35) is Markovian.

Proof: It is easy to see that, for all $k_{\ell}, k_{\ell-1} \in \mathbb{K}$,

$\operatorname{Pr}\left\{C\left(k_{\ell}\right) \mid \Xi\left(k_{\ell}-1\right), \Xi\left(k_{\ell-1}-1\right), \ldots\right\}=\operatorname{Pr}\left\{C\left(k_{\ell}\right) \mid \Xi\left(k_{\ell}-1\right)\right\}$.

The result now follows from (17) and upon noting that $\{\Xi\}_{\mathbb{K}_{0}}$ is Markovian, and $\{A\}_{\mathbb{N}_{0}},\{Q\}_{\mathbb{N}_{0}}$ and $\{R\}_{\mathbb{N}_{0}}$ are deterministic sequences.

Lemma 4: Suppose that Assumption 3 holds and consider

$$
V_{\ell} \triangleq \operatorname{tr} P\left(k_{\ell} \mid k_{\ell}-1\right) \in \mathbb{R}_{\geq 0}, \quad \ell \in \mathbb{N}_{0} .
$$


Then there exists $W \in \mathbb{R}_{\geq 0}$ such that, for all $\ell \in \mathbb{N}_{0}$,

$$
\begin{aligned}
& \mathbf{E}\left\{V_{\ell+1} \mid Z\left(k_{\ell}\right)=(P, i)\right\} \\
& \quad \leq W+\sum_{\delta=1}^{\sigma} \sum_{j \in \mathbb{B}} q_{i j} \psi_{j}(\delta) \mu_{i}\left(k_{\ell}, \delta\right)\left\|\Phi\left(k_{\ell}+\delta, k_{\ell}\right)\right\|^{2} \operatorname{tr} P .
\end{aligned}
$$

Proof: We first note that, given Assumption 3, the holding times $\Delta_{\ell}$ have conditional distribution

$$
\begin{aligned}
& \operatorname{Pr}\left\{\Delta_{\ell}=\delta \mid Z\left(k_{\ell}\right)=(P, i)\right\}=\operatorname{Pr}\left\{\Delta_{\ell}=\delta \mid \Xi\left(k_{\ell}-1\right)=i\right\} \\
& =\sum_{j \in \mathbb{B}} \operatorname{Pr}\left\{\Delta_{\ell}=\delta \mid \Xi\left(k_{\ell}\right)=j, \Xi\left(k_{\ell}-1\right)=i\right\} \\
& \quad \times \operatorname{Pr}\left\{\Xi\left(k_{\ell}\right)=j \mid \Xi\left(k_{\ell}-1\right)=i\right\} \\
& =\sum_{j \in \mathbb{B}} \operatorname{Pr}\left\{\Delta_{\ell}=\delta \mid \Xi\left(k_{\ell}\right)=j\right\} \operatorname{Pr}\left\{\Xi\left(k_{\ell}\right)=j \mid \Xi\left(k_{\ell-1}\right)=i\right\} \\
& =\sum_{j \in \mathbb{B}} q_{i j} \psi_{j}(\delta),
\end{aligned}
$$

thus,

$$
\begin{aligned}
& \mathbf{E}\left\{V_{\ell+1} \mid Z\left(k_{\ell}\right)=(P, i)\right\} \\
& =\sum_{\delta=1}^{\sigma} \sum_{j \in \mathbb{B}} q_{i j} \psi_{j}(\delta) \mathbf{E}\left\{V_{\ell+1} \mid Z\left(k_{\ell}\right)=(P, i), \Delta_{\ell}=\delta\right\} .
\end{aligned}
$$

We next condition on $\varrho\left(k_{\ell}\right)$ defined in (30) to obtain

$$
\begin{gathered}
\mathbf{E}\left\{V_{\ell+1} \mid Z\left(k_{\ell}\right), \Delta_{\ell}\right\} \leq \mathbf{E}\left\{V_{\ell+1} \mid Z\left(k_{\ell}\right), \Delta_{\ell}, \varrho\left(k_{\ell}\right)=1\right\} \\
+\mathbf{E}\left\{V_{\ell+1} \mid Z\left(k_{\ell}\right), \Delta_{\ell}, \varrho\left(k_{\ell}\right)=0\right\} \\
\quad \times \operatorname{Pr}\left\{\varrho\left(k_{\ell}\right)=0 \mid Z\left(k_{\ell}\right), \Delta_{\ell}\right\}
\end{gathered}
$$

and use (31) to write

$$
\begin{aligned}
& \operatorname{Pr}\left\{\varrho\left(k_{\ell}\right)=0 \mid Z\left(k_{\ell}\right)=(P, i), \Delta_{\ell}=\delta\right\} \\
&= \operatorname{Pr}\left\{\varrho\left(k_{\ell}\right)=0 \mid \Xi\left(k_{\ell}-1\right)=i, \Delta_{\ell}=\delta\right\} \\
&= \sum_{j \in \mathbb{B}} \operatorname{Pr}\left\{\varrho\left(k_{\ell}\right)=0 \mid \Xi\left(k_{\ell}\right)=j, \Xi\left(k_{\ell}-1\right)=i, \Delta_{\ell}=\delta\right\} \\
& \quad \times \operatorname{Pr}\left\{\Xi\left(k_{\ell}\right)=j \mid \Xi\left(k_{\ell}-1\right)=i\right\} \\
&= \sum_{j \in \mathbb{B}} q_{i j} \operatorname{Pr}\left\{\varrho\left(k_{\ell}\right)=0 \mid \Xi\left(k_{\ell}\right)=j, \Delta_{\ell}=\delta\right\}=\mu_{i}\left(k_{\ell}, \delta\right) .
\end{aligned}
$$

In what follows, we examine the cases $\varrho\left(k_{\ell}\right) \in\{0,1\}$ separately.

1) For $\varrho\left(k_{\ell}\right)=1$, a suboptimal predictor for $x\left(k_{\ell+1}\right)$ which only uses the received measurements $\{y\}_{k_{\ell}}^{k_{\ell+1}-1} \subset \mathcal{I}\left(k_{\ell+1}-1\right)$ is given by

$$
\begin{gathered}
\check{x}\left(k_{\ell+1}\right)=\Phi\left(k_{\ell+1}, k_{\ell}\right)\left(\mathcal{O}\left(k_{\ell+1}-1, k_{\ell}\right)^{T} \mathcal{O}\left(k_{\ell+1}-1, k_{\ell}\right)\right)^{-1} \\
\times \mathcal{O}\left(k_{\ell+1}-1, k_{\ell}\right)^{T}\left[\begin{array}{c}
y\left(k_{\ell}\right) \\
y\left(k_{\ell+1}\right) \\
\vdots \\
y\left(k_{\ell+1}-1\right)
\end{array}\right] .
\end{gathered}
$$

Since $\{A\}_{\mathbb{N}_{0}},\{Q\}_{\mathbb{N}_{0}}$ and $\{R\}_{\mathbb{N}_{0}}$ are assumed bounded, the matrices $\Phi\left(k_{\ell+1}, k_{\ell}\right)$ and $\mathcal{O}\left(k_{\ell+1}-1, k_{\ell}\right)$ are bounded also. Consequently, the estimation error covariance of $\check{x}\left(k_{\ell+1}\right)$ is bounded. Thus, (due to optimality) in case of the Kalman filter (17), there exists $W_{1} \in \mathbb{R}_{\geq 0}$ such that

$$
\mathbf{E}\left\{V_{\ell+1} \mid Z\left(k_{\ell}\right), \Delta_{\ell}, \varrho\left(k_{\ell}\right)=1\right\} \leq W_{1} .
$$

2) For $\varrho\left(k_{\ell}\right)=0$, the estimation error covariance matrix $P\left(k_{\ell+1} \mid k_{\ell+1}-1\right)$ is upper-bounded by that resulting from the worst case, i.e., where $\gamma_{m}(k)=0, \forall m \in\{1,2, \ldots, M\}$, $\forall k \in\left\{k_{\ell}, k_{\ell}+1, \ldots, k_{\ell+1}-1\right\}$. Use of (17) then gives

$$
\begin{aligned}
\mathbf{E}\left\{V_{\ell+1} \mid Z\left(k_{\ell}\right)=(P, i), \Delta_{\ell}=\delta, \varrho\left(k_{\ell}\right)=0\right\} & \\
\leq & \mathbf{E}\left\{V_{\ell+1} \mid Z\left(k_{\ell}\right)=(P, i), \Delta_{\ell}=\delta, C(k)=0,\right. \\
\left.\forall k \in\left\{k_{\ell}, \ldots, k_{\ell}+\delta-1\right\}\right\} & \operatorname{tr}\left(\begin{array}{rl}
P\left(k_{\ell}+\delta \mid k_{\ell}+\delta-1\right) \mid P\left(k_{\ell} \mid k_{\ell}-1\right)=P, C(k)=0, \\
\left.\quad \forall k \in\left\{k_{\ell}, \ldots, k_{\ell}+\delta-1\right\}\right)
\end{array}\right. \\
= & \operatorname{tr}\left(\Phi\left(k_{\ell}+\delta, k_{\ell}\right) P \Phi\left(k_{\ell}+\delta, k_{\ell}\right)^{T}\right. \\
+ & \left.\sum_{j=1}^{\delta} \Phi\left(k_{\ell}+\delta, k_{\ell}+j\right) Q\left(k_{\ell}+j-1\right) \Phi\left(k_{\ell}+\delta, k_{\ell}+j\right)^{T}\right) .
\end{aligned}
$$

Since $\{A\}_{\mathbb{N}_{0}}$ and $\{Q\}_{\mathbb{N}_{0}}$ are bounded, there exists $W_{2} \in \mathbb{R}_{\geq 0}$ such that

$$
\begin{aligned}
\mathbf{E}\left\{V_{\ell+1} \mid Z\right. & \left.\left(k_{\ell}\right)=(P, i), \Delta_{\ell}=\delta, \varrho\left(k_{\ell}\right)=0\right\} \\
& \leq \operatorname{tr}\left(\Phi\left(k_{\ell}+\delta, k_{\ell}\right) P \Phi\left(k_{\ell}+\delta, k_{\ell}\right)^{T}\right)+W_{2} \\
& =\operatorname{tr}\left(\Phi\left(k_{\ell}+\delta, k_{\ell}\right)^{T} \Phi\left(k_{\ell}+\delta, k_{\ell}\right) P\right)+W_{2} \\
& \leq\left\|\Phi\left(k_{\ell}+\delta, k_{\ell}\right)\right\|^{2} \operatorname{tr} P+W_{2},
\end{aligned}
$$

where we have used [55, Fact 8.12.29].

Substitution of (47)-(49) into (46) provides

$$
\begin{aligned}
\mathbf{E}\left\{V_{\ell+1} \mid\right. & \left.Z\left(k_{\ell}\right)=(P, i), \Delta_{\ell}=\delta\right\} \\
\leq & W_{1}+\mu_{i}\left(k_{\ell}, \delta\right)\left(\left\|\Phi\left(k_{\ell}+\delta, k_{\ell}\right)\right\|^{2} \operatorname{tr} P+W_{2}\right),
\end{aligned}
$$

thus, (45) yields the bound

$$
\begin{array}{r}
\mathbf{E}\left\{V_{\ell+1} \mid Z\left(k_{\ell}\right)=(P, i)\right\} \leq \sum_{\delta=1}^{\sigma} \sum_{j \in \mathbb{B}} q_{i j} \psi_{j}(\delta) \\
\left(W_{1}+\mu_{i}\left(k_{\ell}, \delta\right)\left(\left\|\Phi\left(k_{\ell}+\delta, k_{\ell}\right)\right\|^{2} \operatorname{tr} P+W_{2}\right)\right)
\end{array}
$$

from where the result follows.

To prove Theorem 2, we recall that, by Lemma 4 and (32),

$0 \leq \mathbf{E}\left\{\operatorname{tr} P\left(k_{\ell+1} \mid k_{\ell+1}-1\right) \mid Z\left(k_{\ell}\right)\right\} \leq W+\rho^{\sigma} \operatorname{tr} P\left(k_{\ell} \mid k_{\ell}-1\right)$,

for all $\ell \in \mathbb{N}_{0}$. Thus, following as in the proof of Theorem 1 , it is easy to establish exponential boundedness at the time instants $k_{\ell} \in \mathbb{K}$, i.e., there exist $\alpha_{1}, \beta_{1} \in \mathbb{R}_{\geq 0}$ such that:

$\mathbf{E}\left\{\operatorname{tr} P\left(k_{\ell} \mid k_{\ell}-1\right)\right\} \leq \alpha_{1} \rho^{\ell \sigma}+\beta_{1} \leq \alpha_{1} \rho^{k_{\ell}}+\beta_{1}, \forall k_{\ell} \in \mathbb{K}$,

where we have used the fact that $\Delta_{\ell} \leq \sigma$, thus, $k_{\ell} \leq \ell \sigma$. To establish exponential boundedness at all instants $k \in \mathbb{N}_{0}$, we note that, similar to (49), there exist finite $\alpha_{2} \geq 1, W_{3} \in \mathbb{R}_{\geq 0}$ such that, for all $t \in\left\{0,1, \ldots, \Delta_{\ell}-1\right\}$,

$$
\begin{aligned}
\operatorname{tr} P\left(k_{\ell}+t \mid k_{\ell}+t-1\right) & \leq\left\|\Phi\left(k_{\ell}+t, k_{\ell}\right)\right\|^{2} \operatorname{tr} P\left(k_{\ell} \mid k_{\ell}-1\right)+W_{3} \\
& \leq \alpha_{2} \rho^{t} \operatorname{tr} P\left(k_{\ell} \mid k_{\ell}-1\right)+W_{3} .
\end{aligned}
$$


Taking expectation and using (51) gives

$$
\begin{gathered}
\mathbf{E}\left\{\operatorname{tr} P\left(k_{\ell}+t \mid k_{\ell}+t-1\right)\right\} \leq \alpha_{1} \alpha_{2} \rho^{k_{\ell}+t}+\alpha_{2} \beta_{1} \rho^{t}+W_{3} \\
\leq \alpha_{1} \alpha_{2} \rho^{k_{\ell}+t}+\alpha_{2} \beta_{1}+W_{3}, \quad \forall t \in\left\{0,1, \ldots, \Delta_{\ell}-1\right\} .
\end{gathered}
$$

The latter expression and (51) show exponential boundedness:

$$
\mathbf{E}\{\operatorname{tr} P(k \mid k-1)\} \leq \alpha_{1} \alpha_{2} \rho^{k}+\alpha_{2} \beta_{1}+W_{3}, \quad \forall k \in \mathbb{N}_{0} .
$$

This proves Theorem 2 .

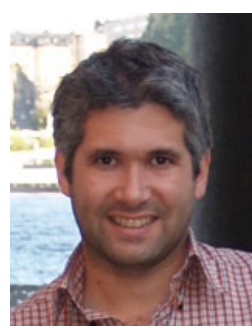

Daniel E. Quevedo (S'97-M'05) received Ingeniero Civil Electrónico and Magister en Ingeniería Electrónica degrees from the Universidad Técnica Federico Santa María, Valparaíso, Chile in 2000. In 2005, he received the Ph.D. degree from The University of Newcastle, Australia, where he is currently an Associate Professor. He has been a visiting researcher at various institutions, including Uppsala University, Sweden, KTH Stockholm, Sweden, Aalborg University, Denmark, Kyoto University, Japan, and INRIA Grenoble, France.

Dr. Quevedo was supported by a full scholarship from the alumni association during his time at the Universidad Técnica Federico Santa María and received several university-wide prizes upon graduating. He received the IEEE Conference on Decision and Control Best Student Paper Award in 2003 and was also a finalist in 2002. In 2009, he was awarded a five-year Australian Research Fellowship. His research interests include several areas of automatic control, signal processing, and power electronics.

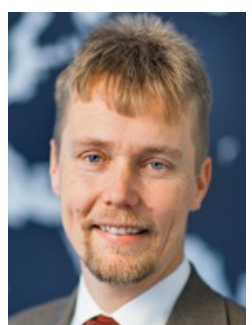

Karl H. Johansson (SM'08) is Director of the KTH ACCESS Linnaeus Centre and Professor at the School of Electrical Engineering, Royal Institute of Technology, Sweden. He is a Wallenberg Scholar and has held a Senior Researcher Position with the Swedish Research Council. He received MSc and $\mathrm{PhD}$ degrees in Electrical Engineering from Lund University. He has held visiting positions at UC Berkeley (1998-2000) and California Institute of Technology (2006-2007). His research interests are in networked control systems, hybrid and embedded control, and control applications in automotive, automation and communication systems. He was a member of the IEEE Control Systems Society Board of Governors 2009 and the Chair of the IFAC Technical Committee on Networked Systems 2008-2011. He has been on the Editorial Boards of Automatica (2003-2006) and IEEE Transactions on Automatic Control (20082010), and is currently on the Editorial Boards of IET Control Theory and Applications and the International Journal of Robust and Nonlinear Control. He was the General Chair of the ACM/IEEE Cyber-Physical Systems Week (CPSWeek) 2010 in Stockholm. He has served on the Executive Committees of several European research projects in the area of networked embedded systems. In 2009, he received the Best Paper Award of the IEEE International Conference on Mobile Ad-hoc and Sensor Systems. He was awarded an Individual Grant for the Advancement of Research Leaders from the Swedish Foundation for Strategic Research in 2005. He received the triennial Young Author Prize from IFAC in 1996 and the Peccei Award from the International Institute of System Analysis, Austria, in 1993. He received Young Researcher Awards from Scania in 1996 and from Ericsson in 1998 and 1999.

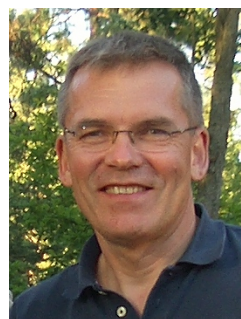

Anders Ahlén (S'80-M'84-SM'90) is full professor and holds the chair in Signal Processing at Uppsala University where he is also the head of the Signals and Systems Division of The Department of Engineering Sciences. He was born in Kalmar, Sweden, and received the $\mathrm{PhD}$ degree in Automatic Control from Uppsala University. He was with the Systems and Control Group, Uppsala University from 1984 1992 as an Assistant and Associate Professor in Automatic Control. During 1991 he was a visiting researcher at the Department of Electrical and Computer Engineering, The University of Newcastle, Australia. He was a visiting professor at the same university in 2008. In 1992 he was appointed Associate Professor of Signal Processing at Uppsala University. During 2001-2004 he was the CEO of Dirac Research AB, a company offering state-of-the-art audio signal processing solutions. He is currently the chairman of the board of the same company. Since 2007 he has been a member of the Uppsala VINN Excellence Center for Wireless Sensor Networks, WISENET. His research interests, which include Signal Processing, Communications and Control, are currently focused on Signal Processing for Wireless Communications, Wireless Systems Beyond 3G, Wireless Sensor Networks, Wireless Control, and Audio Signal Processing.

From 1998 to 2004 he was the Editor of Signal and Modulation Design for the IEEE Transactions on Communications. 